\title{
$r$-Process Nucleosynthesis in the Early Universe Through Fast Mergers of Compact Binaries in Triple Systems
}

\author{
Matteo Bonetti ${ }^{1,2}$, Albino Perego ${ }^{3,4,5,6,7,10}$, Pedro R. Capelo ${ }^{8}$, Massimo Dotti ${ }^{2,4}$ and M. Coleman Miller ${ }^{9}$ \\ ${ }^{1}$ DiSAT, Università degli Studi dell'Insubria, Via Valleggio 11, IT-22100 Como, Italy \\ ${ }^{2}$ INFN, Sezione di Milano-Bicocca, Piazza della Scienza 3, IT-20126 Milano, Italy \\ ${ }^{3}$ INFN, Sezione di Milano-Bicocca, gruppo collegato di Parma, Parco Area delle Scienze 7/A, IT-43124 Parma, Italy \\ ${ }^{4}$ Dipartimento di Fisica G. Occhialini, Università degli Studi di Milano-Bicocca, Piazza della Scienza 3, IT-20126 Milsano, Italy \\ ${ }^{5}$ Dipartimento di Scienze Matematiche Fisiche ed Informatiche, Università di Parma, Parco Area delle Scienze 7/A, IT-43124 Parma, Italy \\ ${ }^{6}$ Institut für Kernphysik, Technische Universität Darmstadt, Schlossgartenstraße 2, DE-64289 Darmstadt, Germany \\ ${ }^{7}$ GSI Helmholtzzentrum für Schwerionenforschung GmbH, Planckstraße 1, DE-64291 Darmstadt, Germany \\ ${ }^{8}$ Center for Theoretical Astrophysics and Cosmology, Institute for Computational Science, University of Zurich, Winterthurerstrasse 190, CH-8057 Zürich, \\ Switzerland \\ ${ }^{9}$ Department of Astronomy and Joint Space-Science Institute, University of Maryland, College Park, MD 20742-2421, USA \\ ${ }^{10}$ Email: albino.perego@mib.infn.it
}

(RECEIVED December 23, 2017; ACCEPTED March 20, 2018)

\begin{abstract}
Surface abundance observations of halo stars hint at the occurrence of $r$-process nucleosynthesis at low metallicity $([\mathrm{Fe} / \mathrm{H}]<-3)$, possibly within the first $10^{8} \mathrm{yr}$ after the formation of the first stars. Possible loci of early-Universe $r$ process nucleosynthesis are the ejecta of either black hole-neutron star or neutron star-neutron star binary mergers. Here, we study the effect of the inclination-eccentricity oscillations raised by a tertiary (e.g. a star) on the coalescence time-scale of the inner compact object binaries. Our results are highly sensitive to the assumed initial distribution of the inner binary semi-major axes. Distributions with mostly wide compact object binaries are most affected by the third object, resulting in a strong increase (by more than a factor of 2) in the fraction of fast coalescences. If instead the distribution preferentially populates very close compact binaries, general relativistic precession prevents the third body from increasing the inner binary eccentricity to very high values. In this last case, the fraction of coalescing binaries is increased much less by tertiaries, but the fraction of binaries that would coalesce within $10^{8} \mathrm{yr}$ even without a third object is already high. Our results provide additional support to the compact-binary merger scenario for $r$-process nucleosynthesis.
\end{abstract}

Keywords: celestial mechanics - gravitation - gravitational waves - ISM: abundances - stars: neutron

\section{INTRODUCTION}

The two main processes responsible for the production of the elements beyond iron group nuclei in the Universe are the rapid and slow neutron capture processes ( $r$-process and $s$ process). The $s$-process occurs in low- to intermediate-mass stars $\left(\lesssim 8 \mathrm{M}_{\odot}\right.$ ) during their asymptotic giant branch phase (e.g. Arlandini et al. 1999; Käppeler et al. 2011; Karakas \& Lattanzio 2014). The duration of the main sequence phase for the stars responsible for the main $s$-process $\left(1.3-3 \mathrm{M}_{\odot}\right)$ sets the expected delay $(\gtrsim 0.6 \mathrm{Gyr})$ for the occurrence of $s$-process nucleosynthesis in the early Universe (e.g. Sneden, Cowan, \& Gallino 2008). The site(s) for the $r$-process nucleosynthesis is (are) still debated, as well as the delay between the formation of the first stars and its first occurrence (see Thielemann et al. 2017, for a recent review).

Observations of the surface abundances of old, metal-poor stars in the galactic halo and in nearby dwarf galaxies hint at the occurrence of $r$-process nucleosynthesis in the very early stages of cosmological evolution (Sneden et al. 2003; Honda et al. 2006; Sneden et al. 2008; Roederer et al. 2014; Ji et al. 2016). The $r$-process occurs when the neutron and photon capture rates are higher than the $\beta$-decay rate of the unstable capturing nuclei. Therefore, $r$-process nucleosynthesis requires special conditions to occur, namely a high neutronto-seed ratio at Nuclear Statistical Equilibrium freeze-out (e.g. Hoffman, Woosley, \& Qian 1997). These conditions are realised for (i) high neutron densities, (ii) expansion timescales shorter than the neutron lifetime (i.e. explosive environments), (iii) neutron-to-proton ratios larger than unity, and (iv) preferentially high-entropy conditions ${ }^{1}$.

\footnotetext{
${ }^{1}$ If $n_{\mathrm{n}}$ and $n_{\mathrm{p}}$ are the neutron and proton densities, respectively, then for $n_{n} /\left(n_{n}+n_{p}\right) \lessgtr 0.25 r$-process nucleosynthesis is also effective in synthesising elements up to the third $r$-process peak for cold, low-entropy matter,
} 
The large scatter in the observed Europium abundance in old metal poor $([\mathrm{Fe} / \mathrm{H}]<-3)$ stars indicates that $r$-process elements must be synthesised in rare and isolated events that inject a significant amount of heavy elements into a relatively small amount of gas. Such gas must undergo star formation before complete elemental mixing has occurred over the entire galaxy. The rare high-yield scenario is also supported by the comparison of plutonium and iron abundances in deepsea sediments (Hotokezaka, Piran, \& Paul 2015). Inhomogeneous galactic chemical evolution models indicate that, in order to explain the distribution of europium abundances at low metallicity, the delay between the first core collapse supernova (CCSN) explosions and the production of $r$-process elements cannot exceed $\sim 10^{8}$ yr (Argast et al. 2004; Cescutti et al. 2015; Wehmeyer, Pignatari, \& Thielemann 2015), if efficient galactic mixing is assumed (see however, van de Voort et al. 2015; Shen et al. 2015; Hirai et al. 2015, for different conclusions based on different modelling and assumptions about the mixing of the ejecta with the interstellar medium).

According to recent models, the necessary conditions for the occurrence of $r$-process nucleosynthesis are not reached in standard CCSNe (e.g. Arcones \& Thielemann 2013, and references therein), whereas magnetically driven CCSNe could potentially enrich the interstellar medium with neutronrich ejecta. These $\mathrm{SNe}$ are expected to be rare and to inject $10^{-4}-10^{-3} \mathrm{M}_{\odot}$ of $r$-process material per SN (Fujimoto, Nishimura, \& Hashimoto 2008; Winteler et al. 2012; Nishimura, Takiwaki, \& Thielemann 2015). The presence of rapidly rotating stellar cores, which are needed for these explosions, is more likely realised at lower metallicity (Woosley $\&$ Heger 2006) and suggests a possible connection with hypernovae and long gamma-ray bursts. Unfortunately, details of the magnetically driven CCSN explosion mechanism and even the existence of such explosions are still debated (e.g. Mösta et al. 2014).

Another possible site for $r$-process nucleosynthesis in the Universe are compact-binary mergers (CBMs), with at least one binary component being a neutron star (NS) (Lattimer \& Schramm 1974; Symbalisty \& Schramm 1982; Eichler et al. 1989; Freiburghaus, Rosswog, \& Thielemann 1999). This long-standing conjecture has been recently confirmed by the combined electromagnetic and gravitational wave $(\mathrm{GW})$ detection from a likely binary NS merger (e.g. Abbott et al. 2017b, 2017a; Pian et al. 2017; Tanvir et al. 2017; Coulter et al. 2017; Nicholl et al. 2017; Chornock et al. 2017). The electromagnetic signal is compatible with a kilonova emission, which is thought to be powered by the radioactive decay of the freshly synthesised $r$-process elements (e.g. Rosswog 2015; Fernández \& Metzger 2016; Metzger 2017, for recent reviews). CBMs can eject $10^{-4}-10^{-2} \mathrm{M}_{\odot}$ per merger event in the form of dynamical, viscous, neutrino-driven, or magnetically driven ejecta, although the precise amount of ejecta depends on the intrinsic properties of the merging binary, as

i.e. $s \lesssim 20 k_{\mathrm{B}} /$ baryon, where $k_{\mathrm{B}}$ is the Boltzmann constant (see, e.g. Martin et al. 2017). well as on the still unknown properties of the nuclear equation of state above nuclear saturation density (see, e.g. Surman et al. 2008; Korobkin et al. 2012; Hotokezaka et al. 2013; Fernández \& Metzger 2013; Bauswein, Goriely, \& Janka 2013; Wanajo et al. 2014; Perego et al. 2014; Foucart et al. 2015; Martin et al. 2015; Just et al. 2015; Wu et al. 2016; Radice et al. 2016; Roberts et al. 2017; Bovard et al. 2017, for some recent discussions).

CBMs are driven by the emission of GWs. However, the corresponding merger time-scale in an isolated binary depends strongly on the initial orbital parameters of the compact binary. Fast (i.e. within $10^{8} \mathrm{yr}$ ) binary mergers require small orbital separations and/or high eccentricities (Peters 1964). For this reason, the possibility for CBMs to be a viable site for the $r$-process nucleosynthesis in the early Universe is still disputed.

The strong constraints on the initial semi-major axis and eccentricity for there to be fast coalescence are relaxed if the binary interacts with other objects. The occurrence of such triple or multiple systems is not negligible: a significant fraction of massive stars $\left(M \gtrsim 8 \mathrm{M}_{\odot}\right.$, whose $\mathrm{SN}$ explosion produces an NS or black hole-BH-remnant) are bound in multiple systems (e.g. Duchêne \& Kraus 2013). In the presence of a third object, the stellar system can undergo KozaiLidov (KL) oscillations (Kozai 1962; Lidov 1962), in which the eccentricity and inclination of the inner binary oscillate with periods significantly longer than the inner orbital period. Depending on the triplet configuration, the inner binary can increase its eccentricity significantly, which then decreases the time to coalescence due to $\mathrm{GW}$ emission.

The effects of the KL mechanism have been invoked in many different astrophysical contexts including planetary dynamics (Holman, Touma, \& Tremaine 1997; Ford, Kozinsky, \& Rasio 2000; Katz, Dong, \& Malhotra 2011; Naoz, Farr, \& Rasio 2012; Naoz et al. 2013), interactions of stellar size objects in globular clusters (Antonini et al. 2016; Antognini $\&$ Thompson 2016) and around massive BHs (Antonini \& Perets 2012; VanLandingham et al. 2016), and triple massive BH systems (Miller \& Hamilton 2002; Blaes, Lee, \& Socrates 2002; Iwasawa, Funato, \& Makino 2006; Hoffman \& Loeb 2007; Kulkarni \& Loeb 2012; Bonetti et al. 2016).

In a previous work similar in spirit, Thompson (2011) showed that the rate of CBMs can be significantly enhanced by the KL mechanism within a Hubble time. In this paper, we explore under which conditions the KL mechanism can affect the dynamics of a triplet hosting an inner compact binary, such that the coalescence time-scale becomes shorter than 100 Myr.

The paper is structured as follows. In Section 2, we introduce the parameters involved in our calculations, perform basic estimates, and present the most relevant time-scales. We present the equations that describe the triplet evolution in the secular approximation in Section 3. Section 4 is devoted to the analysis of the evolution of the inner compact binary in a few selected cases, whereas in Section 5, the effect of the $\mathrm{KL}$ mechanism on compact binary populations is explored. 

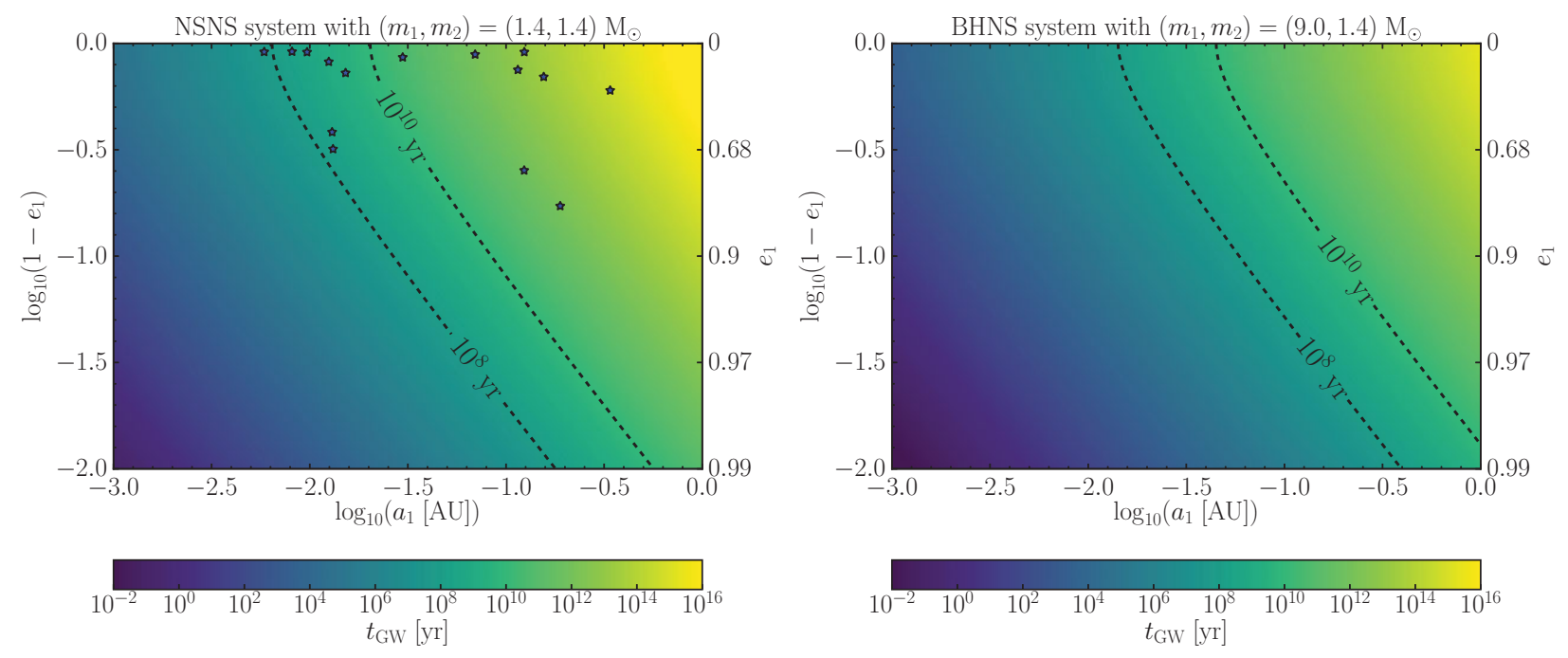

Figure 1. Merger time-scale of an isolated binary due to emission of GWs, as a function of the initial semi-major axis $a_{1}$ and eccentricity $e_{1}$. Left panel: NS binary with masses $m_{1}=m_{2}=1.4 \mathrm{M}_{\odot}$. Blue stars refer to the measured or estimated orbital properties of observed NSNS systems (see Table 1 for more details). Right panel: BHNS binary with masses $m_{1}=9 \mathrm{M}_{\odot}$ and $m_{2}=1.4 \mathrm{M}_{\odot}$. Dashed lines mark the values of semi-major axis and eccentricity for which the coalescence takes place within $10^{8}$ and $10^{10} \mathrm{yr}$.

Finally, we discuss our results and conclude in Section 6. In Appendix A, we summarise and discuss the results of our extensive parameter space exploration.

\section{PRELIMINARY ESTIMATES AND TIME-SCALES}

For an isolated binary system, the merger time-scale is given by the gravitational radiation time, $t_{\mathrm{GW}}$, obtained by integrating the coupled evolution of the semi-major axis and of the inner eccentricity (see, e.g. Peters 1964). If $m_{1}$ and $m_{2}$ (with $\left.q \equiv m_{2} / m_{1} \leqslant 1\right)$ are the masses of the two bodies orbiting each other and emitting GWs,

$$
\begin{aligned}
t_{\mathrm{GW}}= & 3.2452 \times 10^{8} \mathrm{yr}\left(\frac{a_{1}}{0.01 \mathrm{AU}}\right)^{4} \\
& \left(\frac{\mu_{\mathrm{CB}}}{\mathrm{M}_{\odot}}\right)^{-1}\left(\frac{m_{1}+m_{2}}{5 \mathrm{M}_{\odot}}\right)^{-2} f\left(e_{1}\right),
\end{aligned}
$$

where $a_{1}$ is the semi-major axis of the initial orbit, $e_{1}$ its eccentricity, $\mu_{\mathrm{CB}}=m_{1} m_{2} /\left(m_{1}+m_{2}\right)$ the reduced mass of the inner compact binary, and $f\left(e_{1}\right)$ is a sensitive function of the initial eccentricity:

$$
\begin{aligned}
f\left(e_{1}\right)= & {\left[\frac{1-e_{1}^{2}}{e_{1}^{12 / 19}}\left(1+\frac{121}{304} e_{1}^{2}\right)^{-870 / 2299}\right]^{4} } \\
& \times \int_{0}^{e_{1}} \mathrm{~d} \bar{e} \frac{\bar{e}^{29 / 19}}{\left(1-\bar{e}^{2}\right)^{3 / 2}}\left(1+\frac{121}{304} \bar{e}^{2}\right)^{1181 / 2299} .
\end{aligned}
$$

Following Peters (1964), expansions of $f(e)$ can be computed for $e_{1} \rightarrow 0, f\left(e_{1}\right) \approx(19 / 48)\left[\left(1-e_{1}^{2}\right)\left(1+121 e_{1}^{2} / 304\right)\right]^{4}$, and for $e_{1} \rightarrow 1, f\left(e_{1}\right) \approx(304 / 425)\left(1-e_{1}^{2}\right)^{7 / 2}$. We find that a good approximation over the whole range of $e_{1}$ is provided by $f\left(e_{1}\right)=\left(1-e_{1}^{2}\right)^{\left(8-e_{1}\right) / 2} g\left(e_{1}\right)$, where $g\left(e_{1}\right)$ is a monotonically increasing function varying between $g(0)=19 / 48$ and $g(1)$ $=304 / 425^{2}$.

In Figure 1, we present the GW time-scale [equation (1)] as a function of $a_{1}$ and $e_{1}$ for a typical binary NS (NSNS) system characterised by $m_{1}=m_{2}=1.4 \mathrm{M}_{\odot}$ (left panel) and for a black hole-NS (BHNS) binary system with $m_{1}=9 \mathrm{M}_{\odot}$ and $m_{2}=1.4 \mathrm{M}_{\odot}$ (right panel). Clearly, $t_{\mathrm{GW}}$ depends strongly on the orbital parameters. In the case of binary NS systems, we report also the orbital properties of the observed NSNS systems (see Tauris et al. 2017, and also Table 1). Due to the narrow distributions of NS masses in NSNS systems, the calculation of $t_{\mathrm{GW}}$ for our reference case $\left(m_{1}=m_{2}=\right.$ $1.4 \mathrm{M}_{\odot}$ ) provides an accurate enough estimate also for the merger time-scales of the observed sample of NSNS binaries. Amongst the observed systems, $t_{\mathrm{GW}}$ is $<10^{8} \mathrm{yr}$ in only one case, whereas many systems will not coalesce within a Hubble time. A fast merger time-scale (of the order of or below $10^{8} \mathrm{yr}$ ) requires a small orbit, $a_{1} \lesssim 0.01 \mathrm{AU}$, or at larger separations $\left(a_{1} \sim 0.2 \mathrm{AU}\right)$ a very high eccentricity, $e_{1}$ $\gtrsim 0.99$. Due to the larger mass of the $\mathrm{BH}$, the $\mathrm{GW}$ time-scale is significantly smaller for BHNS systems at a fixed separation. However, fast mergers still require small orbits or high eccentricities. The lack of observations for such systems prevents a direct comparison with orbital configurations realised in nature.

If the compact binary is part of a gravitationally bound triple system, its properties are fully specified once the positions, velocities, and masses of the three bodies are known at one instant in time. We restrict our study to the case where the

\footnotetext{
${ }^{2}$ A hyperbolic fit $g(x)=0.38+1 /[49.3(-x+1.08)]$ provides an expression accurate to within $1 \%$ between $0<x<0.99$.
} 
Table 1. Properties of the observed NSNS systems (adapted from Tauris et al. 2017). Pulsar name indicates the name of the radio pulsar(s) in the system. Quantities in brackets are assumed. In particular, if $m_{2}$ is not measured, but $m_{1}+m_{2}$ is, $m_{2}=1.28 \mathrm{M}_{\odot}$ is assumed (central value of the measured secondary mass distribution; for B1930-1852, $m_{2}=1.29 \mathrm{M}_{\odot}$ to be compatible with observational limits). If also $m_{1}+m_{2}$ is not measured, $m_{1}+m_{2}=2.725 \mathrm{M}_{\odot}$ is assumed (central value of the measured total mass distribution). The semi-major axis $a_{1}$ is computed assuming a Keplerian orbit. In the location column, GF and GC stand for galactic field and globular cluster, respectively.

\begin{tabular}{|c|c|c|c|c|c|c|c|c|}
\hline Pulsar name & $\begin{array}{c}T_{\text {orb }} \\
{[\mathrm{d}]}\end{array}$ & $\begin{array}{c}e_{1} \\
{[-]}\end{array}$ & $\begin{array}{c}m_{1} \\
{\left[\mathrm{M}_{\odot}\right]}\end{array}$ & $\begin{array}{c}m_{2} \\
{\left[\mathrm{M}_{\odot}\right]}\end{array}$ & $\begin{array}{c}m_{1}+m_{2} \\
{\left[\mathrm{M}_{\odot}\right]}\end{array}$ & $\begin{array}{c}a_{1} \\
{\left[10^{-2} \mathrm{AU}\right]}\end{array}$ & Location & $\begin{array}{l}t_{\mathrm{GW}} \\
{[\mathrm{yr}]}\end{array}$ \\
\hline J0453+1559 & 4.072 & 0.113 & 1.559 & 1.774 & 2.734 & 6.959 & GF & $1.44 \times 10^{12}$ \\
\hline J0737-3039 & 0.102 & 0.088 & 1.338 & 1.249 & 2.587 & 0.586 & GF & $8.51 \times 10^{7}$ \\
\hline $\mathrm{J} 1518+4904$ & 8.634 & 0.249 & (1.428) & $(1.28)$ & 2.718 & 11.49 & GF & $\left(8.67 \times 10^{12}\right)$ \\
\hline B1534+12 & 0.421 & 0.274 & 1.346 & 1.333 & 2.678 & 1.522 & GF & $2.71 \times 10^{9}$ \\
\hline J1753-2240 & 13.638 & 0.304 & (1.445) & $(1.28)$ & $(2.725)$ & $(15.562)$ & GF & $\left(2.63 \times 10^{13}\right)$ \\
\hline $\mathrm{J} 1755-2550$ & 9.696 & 0.089 & (1.445) & $(1.28)$ & $(2.725)$ & $(12.40)$ & GF & $\left(1.46 \times 10^{13}\right)$ \\
\hline J1756-2251 & 0.320 & 0.181 & 1.341 & 1.230 & 2.570 & 1.250 & GF & $1.64 \times 10^{9}$ \\
\hline J1811-1736 & 18.779 & 0.828 & $<1.64(1.29)$ & $>0.93(1.28)$ & 2.57 & 18.89 & GF & $\left(1.78 \times 10^{12}\right)$ \\
\hline $\mathrm{J} 1829+2456$ & 1.176 & 0.139 & $<1.38(1.31)$ & $>1.22(1.28)$ & 2.59 & 2.976 & GF & $\left(5.40 \times 10^{10}\right)$ \\
\hline J1906+0746 & 0.166 & 0.085 & 1.291 & 1.322 & 2.613 & 0.812 & GF & $3.05 \times 10^{8}$ \\
\hline $\mathrm{J} 1913+1102$ & 0.206 & 0.090 & $<1.84(1.60)$ & $>1.04(1.28)$ & 2.88 & 0.969 & GF & $\left(4.65 \times 10^{8}\right)$ \\
\hline B1913+16 & 0.323 & 0.617 & 1.440 & 1.389 & 2.828 & 1.299 & GF & $2.98 \times 10^{8}$ \\
\hline B1930-1852 & 45.060 & 0.399 & $>1.30(1.30)$ & $<1.32(1.29)$ & 2.59 & 33.94 & GF & $\left(5.26 \times 10^{14}\right)$ \\
\hline B $1807-2500 B$ & 9.957 & 0.747 & 1.366 & 1.206 & 2.572 & 12.38 & GC & $1.03 \times 10^{12}$ \\
\hline $\mathrm{B} 2127+11 \mathrm{C}$ & 0.335 & 0.681 & 1.358 & 1.354 & 2.713 & 1.314 & GC & $2.14 \times 10^{8}$ \\
\hline
\end{tabular}

triplet is hierarchical and its evolution is well described by a secular approach. Under these hypotheses, the description of the triplet is simplified because it can be treated as consisting of the following two distinct, but coupled, binary systems:

(i) An inner binary, which in our case is always represented by a compact binary and is characterised by the following minimal set of six parameters:

- $a_{1}$, the inner semi-major axis, such that $5 \times 10^{-3} \mathrm{AU}$ $<a_{1}<0.3 \mathrm{AU}$, which is compatible with the observed NSNS semi-major axes. We also include the possibility that $a_{1}$ is smaller than what is currently observed, because a population of tight compact binaries could be difficult to observe, due to the short $t_{\mathrm{GW}}$.

- $e_{1}$, the inner eccentricity, such that $0<e_{1}<1$.

- The primary and secondary masses, $m_{1}$ and $m_{2}$. For NSs, we consider $1.0 \mathrm{M}_{\odot}<m_{\mathrm{NS}}<2.4 \mathrm{M}_{\odot}$, which is $\sim 20 \%$ wider than the maximum and minimum observed NS masses; for BHs, we choose $5 \mathrm{M}_{\odot}<m_{\mathrm{BH}}$ $<30 \mathrm{M}_{\odot}$, which is within the highly uncertain range of stellar BH masses observed in binaries.

- The inner argument of the pericentre, $g_{1}$, which locates the angular position of the pericentre in the orbital plane and is between 0 and $2 \pi$ radians (see left panel of Figure 2).

- The inner inclination angle, $i_{1}$, which is the angle between the positive $z$ direction and the orbital angular momentum of the inner binary, $\mathbf{G}_{1}$, i.e. $\cos i_{1}=$ $\mathbf{G}_{1} \cdot \hat{\mathbf{z}} / G_{1}$, where $\hat{\mathbf{z}}$ is the unitary positive vector along $z$ (where we define $z$ to be along the direction of the total angular momentum, $\mathbf{H}=\mathbf{G}_{1}+\mathbf{G}_{2}=H \hat{\mathbf{z}}$ ). Thus, in general, $0 \leqslant i_{1} \leqslant \pi$ and $i_{1}<\pi / 2$ represents counter-clockwise motion (see right panel of Figure 2).

(ii) An outer binary system, in which the inner binary is treated as a point of mass $m_{1}+m_{2}$, located in its centre of mass, and the second component is a main sequence star of mass $m_{3}$. The outer binary is characterised by a set of five parameters, similar to that of the inner binary:

- $a_{2}$, the outer semi-major axis, such that $3 \times 10^{-2}$ $\mathrm{AU}<a_{1}<10 \mathrm{AU}$. Observed external semi-major axes of hierarchical triple stellar systems span a wide range of values, going from a fraction of AU up to thousands of AU. We impose an upper limit of $10 \mathrm{AU}$ to ensure a significant coupling between the inner and the outer binary.

- $e_{2}$, the outer eccentricity, such that $0<e_{2}<1$.

- The tertiary mass, $m_{3}$, with $3 \mathrm{M}_{\odot}<m_{3}<15 \mathrm{M}_{\odot}$. The lower limit on $m_{3}$ is required to have an adequate gravitational influence on the dynamics of the inner binary, whose total mass is always above $2 \mathrm{M}_{\odot}$. Our choice is also supported by the fact that stars in the early Universe are metal-poor and therefore more massive (e.g. Bromm, Coppi, \& Larson 2002). Moreover, hierarchical triplets with light tertiary masses are easier to unbind by external perturbations. The upper limit is related to the stability of the triplet itself. Indeed, the presence of a main sequence star 

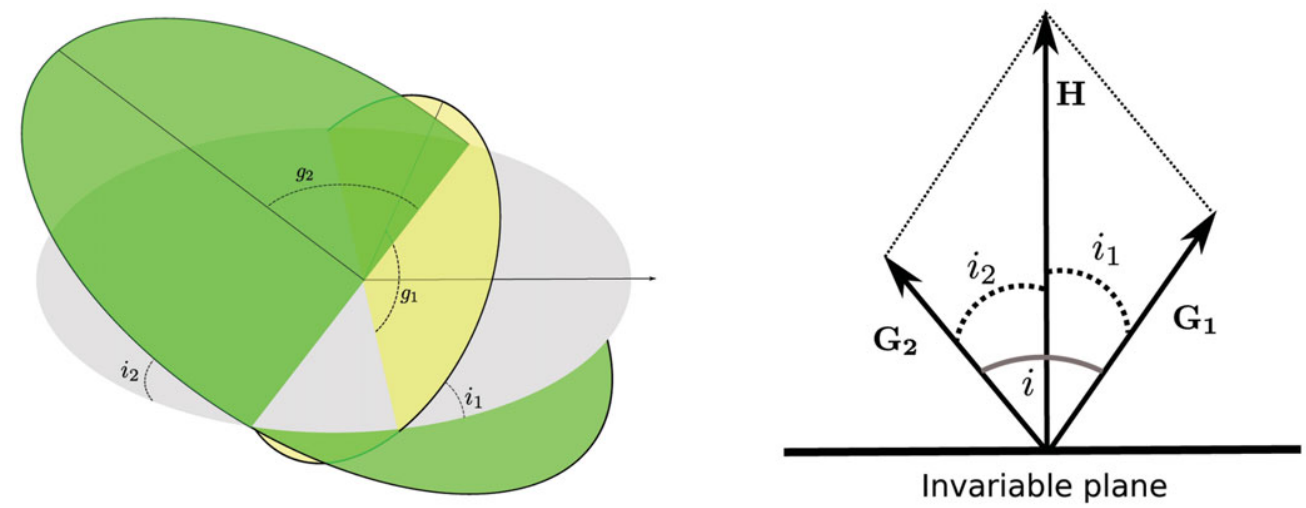

Figure 2. Schematic description of the configuration of hierarchical triplets. Left panel: configuration in the 3D space. Right panel: configuration of the angular momenta. Note that the definition of the relative inclination $i \equiv i_{1}+i_{2}$ results rather natural.

requires consideration of the stellar main-sequence lifetime:

$$
t_{\mathrm{MS}} \sim 10^{10} \mathrm{yr}\left(\frac{m_{3}}{\mathrm{M}_{\odot}}\right)^{-5 / 2} .
$$

For durations greater than $t_{\mathrm{MS}}$, the formation of a white dwarf or the explosion of the star as a CCSN can significantly alter the properties of the triplet or even destroy it. Since we are interested in time intervals less than $10^{8} \mathrm{yr}$, we use an upper limit for $m_{3}$ such that $t_{\mathrm{MS}}$ equals $10^{7} \mathrm{yr}$, i.e. $10 \%$ of the maximum allowed time. This corresponds roughly to $16 \mathrm{M}_{\odot}$, we also notice that $t_{\mathrm{MS}} \sim 10^{8} \mathrm{yr}$ corresponds to $m_{3} \approx 6.3 \mathrm{M}_{\odot}$.

- The outer argument of the pericentre, $g_{2}$, which like $g_{1}$ can vary over $2 \pi$ (see left panel of Figure 2).

- The outer inclination angle, $i_{2}$, analogous to $i_{1}$, but for the outer orbit: $\cos i_{2}=\mathbf{G}_{2} \cdot \hat{\mathbf{z}} / G_{2}$, where $\mathbf{G}_{2}$ is the orbital angular momentum of the outer binary (see right panel of Figure 2).

The only relevant inclination angle is the relative angle between the inner and the outer binaries, $i \equiv i_{2}+i_{1}$. Hence, the hierarchical triplet is characterised by a set of 10 independent parameters.

The hierarchical nature of the triplet and the validity of our secular approach constrain the values of the allowed orbital parameters. In particular, we require that our triplets satisfy the stability criterion reported by Mardling \& Aarseth (2001):

$$
\frac{a_{2}}{a_{1}}>2.8\left(1+\frac{m_{3}}{m_{1}+m_{2}}\right)^{2 / 5} \frac{\left(1+e_{2}\right)^{2 / 5}}{\left(1-e_{2}\right)^{6 / 5}} .
$$

This relation was obtained for purely Newtonian coplanar prograde orbits of the inner and outer binaries. Inclined and retrograde orbits are expected to be more stable (Mardling $\&$ Aarseth 2001) $)^{3}$, so equation (4) provides a conservative stability limit. We assume that triplets for which equation (4)

\footnotetext{
${ }^{3}$ For misaligned orbits, the critical outer semi-major axis for which a triplet remains stable can be reduced by a factor $k=1-0.3 i / \pi$ (see Mardling \&
}

is not satisfied cannot be treated with the secular approximation and enter the chaotic regime. The precise evolution of such systems requires direct integration of the equations of motion for the three bodies (see, e.g. Hoffman \& Loeb 2007; Antonini et al. 2016; Bonetti et al. 2016, and references therein). In the following, we will assume that in those cases, the triplet usually gets disrupted and that the more massive third body probably replaces the lighter NS in the inner binary. Thus, those systems will never host a CBM.

A hierarchical triplet is potentially subject to a large variety of effects that influence its dynamics (Heggie 1975). Assuming that the triple system is not influenced by dynamical interactions with other external bodies, the most important effects are the general relativistic (GR) precession of the inner periastron and the KL mechanism. The GR precession forces the argument of pericentre of a binary to monotonically increase from 0 to $2 \pi$, i.e. the ellipse rotates in the orbital plane and describes rosetta-like orbits, on a time-scale that is given approximately by (Miller \& Hamilton 2002; Blaes et al. 2002)

$$
\begin{aligned}
t_{\mathrm{GR}, \mathrm{prec}} & \sim 30 \mathrm{yr}\left(\frac{m_{1}+m_{2}}{5 \mathrm{M}_{\odot}}\right)^{-3 / 2} \\
& \left(\frac{a_{1}}{0.01 \mathrm{AU}}\right)^{5 / 2}\left(1-e_{1}^{2}\right) .
\end{aligned}
$$

If the mutual inclination angle $i$ is large enough, the KL mechanism can induce an oscillation in the inner eccentricity. If we consider the limit ${ }^{4} m_{2} \rightarrow 0$ and the first non-vanishing contribution (i.e. the quadrupole term) in the $a_{1} / a_{2}$ expansion of the equations of motion, we obtain the classical KL mechanism and $e_{1}$ oscillates up to a maximum value given by

$$
e_{1, \max } \approx\left(1-\frac{5}{3} \cos ^{2} i\right)^{1 / 2}
$$

Aarseth 2001, and reference therein). Note that the minimum allowed $a_{2}$ is achieved for coplanar retrograde systems.

${ }^{4}$ This condition actually means that the total angular momentum of the system is dominated by the outer binary. 
on a characteristic time-scale

$$
\begin{aligned}
t_{\mathrm{KL}, \text { quad }} \sim 0.4 \mathrm{yr}\left(\frac{a_{1}}{0.01 \mathrm{AU}}\right)^{-3 / 2}\left(\frac{m_{1}+m_{2}}{5 \mathrm{M}_{\odot}}\right)^{1 / 2} \\
\left(\frac{m_{3}}{10 \mathrm{M}_{\odot}}\right)^{-1}\left(1-e_{2}^{2}\right)^{3 / 2}\left(\frac{a_{2}}{0.1 \mathrm{AU}}\right)^{3} .
\end{aligned}
$$

If $t_{\mathrm{GR} \text {, prec }} \lesssim t_{\mathrm{KL} \text {, quad }}$, the GR precession can erase the KL resonance because it destroys the coherent piling up of the perturbation induced by the third body. Because of the GR precession, the maximum eccentricity reached can be much lower (Miller \& Hamilton 2002). Using equations (5) and (7), we obtain a criterion on the orbital parameters for the KL mechanism to be efficient against the GR precession:

$$
\begin{gathered}
a_{2}<0.53 \mathrm{AU}\left(\frac{a_{1}}{0.01 \mathrm{AU}}\right)^{4 / 3}\left(\frac{m_{1}+m_{2}}{5 \mathrm{M}_{\odot}}\right)^{-1 / 3} \\
\left(\frac{m_{3}}{m_{1}+m_{2}}\right)^{1 / 3}\left(\frac{1-e_{1}^{2}}{1-e_{2}^{2}}\right)^{1 / 2} \cdot
\end{gathered}
$$

If the KL resonance is not suppressed, the octupole term in the $a_{1} / a_{2}$ expansion modulates the $e_{1}$ oscillation, on a longer time-scale given by

$$
\begin{gathered}
t_{\mathrm{KL}, \text { oct }} \sim 5.3 \mathrm{yr}\left(\frac{a_{1}}{0.01 \mathrm{AU}}\right)^{-5 / 2}\left(\frac{m_{1}+m_{2}}{5 \mathrm{M}_{\odot}}\right)^{3 / 2} \\
\left(\frac{m_{3}}{10 \mathrm{M}_{\odot}}\right)^{-1} \frac{\left(1-e_{2}^{2}\right)^{5 / 2}}{e_{2}}\left(\frac{a_{2}}{0.1 \mathrm{AU}}\right)^{4} \\
\left(\frac{\left|m_{1}-m_{2}\right|}{1 \mathrm{M}_{\odot}}\right)^{-1} .
\end{gathered}
$$

The effect of the octupole modulation is to increase $e_{1, \max }$.

\section{SECULAR EVOLUTION OF ISOLATED HIERARCHICAL TRIPLETS}

The evolution of the orbital elements of the inner $\left(a_{1}, e_{1}\right.$, and $\left.g_{1}\right)$ and outer $\left(e_{2} \text { and } g_{2}\right)^{5}$ binaries is obtained under two approximations: $(i)$ the properties of each binary are orbitally averaged, and (ii) the equations of motion are approximated with their expansion up to the second order (octupole term) in $a_{1} / a_{2}$. In detail, we follow Blaes et al. (2002) by integrating the following differential equations:

$$
\begin{gathered}
\frac{d a_{1}}{d t}=-\frac{64 G^{3} m_{1} m_{2}\left(m_{1}+m_{2}\right)}{5 c^{5} a_{1}^{3}\left(1-e_{1}^{2}\right)^{7 / 2}}\left(1+\frac{73}{24} e_{1}^{2}+\frac{37}{96} e_{1}^{4}\right) \\
\frac{d g_{1}}{d t}=6 C_{2}\left\{\frac{1}{G_{1}}\left[4 \cos i^{2}+\left(5 \cos 2 g_{1}-1\right)\left(1-e_{1}^{2}-\cos ^{2} i\right)\right]\right. \\
\left.+\frac{\cos i}{G_{2}}\left[2+e_{1}^{2}\left(3-5 \cos 2 g_{1}\right)\right]\right\}+C_{3} e_{2} e_{1}\left(\frac{1}{G_{2}}+\frac{\cos i}{G_{1}}\right) \\
\left\{\sin g_{1} \sin g_{2}\left[A+10\left(3 \cos ^{2} i-1\right)\left(1-e_{1}^{2}\right)\right]-5 \cos i B \cos \phi\right\} \\
\quad-C_{3} e_{2} \frac{1-e_{1}^{2}}{e_{1} G_{1}}\left[10 \cos i\left(1-\cos ^{2} i\right)\left(1-3 e_{1}^{2}\right) \sin g_{1} \sin g_{2}\right. \\
\left.+\cos \phi\left(3 A-10 \cos ^{2} i+2\right)\right] \\
+\frac{3}{c^{2} a_{1}\left(1-e_{1}^{2}\right)}\left[\frac{G\left(m_{1}+m_{2}\right)}{a_{1}}\right]^{3 / 2},
\end{gathered}
$$

\footnotetext{
${ }^{5}$ Here, we are neglecting the effect of GW emission on the shrinking of the outer binary, hence $a_{2}$ remains constant throughout the integration.
}

$$
\begin{aligned}
\frac{\mathrm{d} e_{1}}{\mathrm{~d} t}= & 30 C_{2} \frac{e_{1}\left(1-e_{1}^{2}\right)}{G_{1}}\left(1-\cos ^{2} i\right) \sin 2 g_{1} \\
& -C_{3} e_{2} \frac{1-e_{1}^{2}}{G_{1}}\left[35 \cos \phi\left(1-\cos ^{2} i\right) e_{1}^{2} \sin 2 g_{1}\right. \\
& -10 \cos i\left(1-e_{1}^{2}\right)\left(1-\cos ^{2} i\right) \cos g_{1} \sin g_{2} \\
& \left.-A\left(\sin g_{1} \cos g_{2}-\cos i \cos g_{1} \sin g_{2}\right)\right] \\
& -\frac{304 G^{3} m_{1} m_{2}\left(m_{1}+m_{2}\right) e_{1}}{15 c^{5} a_{1}^{4}\left(1-e_{1}^{2}\right)^{5 / 2}}\left(1+\frac{121}{304} e_{1}^{2}\right),
\end{aligned}
$$

$$
\begin{aligned}
\frac{\mathrm{d} g_{2}}{\mathrm{~d} t}= & 3 C_{2}\left\{\frac{2 \cos i}{G_{1}}\left[2+e_{1}^{2}\left(3-5 \cos 2 g_{1}\right)\right]\right. \\
& \left.+\frac{1}{G_{2}}\left[4+6 e_{1}^{2}+\left(5 \cos ^{2} i-3\right)\left(2+3 e_{1}^{2}-5 e_{1}^{2} \cos 2 g_{1}\right)\right]\right\} \\
& -C_{3} e_{1} \sin g_{1} \sin g_{2}\left\{\frac{4 e_{2}^{2}+1}{e_{2} G_{2}} 10 \cos i\left(1-\cos ^{2} i\right)\left(1-e_{1}^{2}\right)\right. \\
& \left.-e_{2}\left(\frac{1}{G_{1}}+\frac{\cos i}{G_{2}}\right)\left[A+10\left(3 \cos ^{2} i-1\right)\left(1-e_{1}^{2}\right)\right]\right\} \\
& -C_{3} e_{1} \cos \phi\left[5 B \cos i e_{2}\left(\frac{1}{G_{1}}+\frac{\cos i}{G_{2}}\right)+\frac{4 e_{2}^{2}+1}{e_{2} G_{2}} A\right],
\end{aligned}
$$

$$
\begin{aligned}
\frac{\mathrm{d} e_{2}}{\mathrm{~d} t}= & C_{3} e_{1} \frac{1-e_{2}^{2}}{G_{2}}\left[10 \cos i\left(1-\cos ^{2} i\right)\left(1-e_{1}^{2}\right) \sin g_{1} \cos g_{2}\right. \\
& \left.+A\left(\cos g_{1} \sin g_{2}-\cos i \sin g_{1} \cos g_{2}\right)\right],
\end{aligned}
$$

where $\phi$ is the angle between the periastron directions,

$$
\cos \phi=-\cos g_{1} \cos g_{2}-\cos i \sin g_{1} \sin g_{2},
$$

and the cosine of the mutual inclination of the binaries can be expressed as a function of the magnitudes of the angular momenta of the inner binary $\left(G_{1}=m_{1} m_{2}\left\{\left[G a_{1}\left(1-e_{1}^{2}\right)\right] /\left[m_{1}+\right.\right.\right.$ $\left.\left.\left.m_{2}\right]\right\}^{1 / 2}\right)$, of the outer binary $\left(G_{2}=m_{3}\left(m_{1}+m_{2}\right)\left\{\left[G a_{2}(1-\right.\right.\right.$ $\left.\left.\left.\left.e_{2}^{2}\right)\right] /\left[m_{1}+m_{2}+m_{3}\right]\right\}^{1 / 2}\right)$, and of the whole triple system $\left(H=G_{1} \cos i_{1}+G_{2} \cos i_{2}\right)$ as follows:

$$
\cos i=\frac{H^{2}-G_{1}^{2}-G_{2}^{2}}{2 G_{1} G_{2}} .
$$

The closure of the system of differential equations is obtained through the angular momentum evolution equation:

$$
\begin{gathered}
\frac{d H}{d t}=-\frac{32 G^{3} m_{1}^{2} m_{2}^{2}}{5 c^{5} a_{1}^{3}\left(1-e_{1}^{2}\right)^{2}}\left[\frac{G\left(m_{1}+m_{2}\right)}{a_{1}}\right]^{1 / 2} \\
\left(1+\frac{7}{8} e_{1}^{2}\right) \frac{G_{1}+G_{2} \cos i}{H} .
\end{gathered}
$$

In equations (11)-(14), $A=4+3 e_{1}^{2}-5\left(1-\cos ^{2} i\right) B / 2$ and $B=2+5 e_{1}^{2}-7 e_{1}^{2} \cos 2 g_{1}$, whereas the quantities $C_{2}$ 
and $C_{3}$ (defined as in Ford et al. 2000),

$$
\begin{aligned}
C_{2} & =\frac{G m_{1} m_{2} m_{3}}{16\left(m_{1}+m_{2}\right) a_{2}\left(1-e_{2}^{2}\right)^{3 / 2}}\left(\frac{a_{1}}{a_{2}}\right)^{2}, \\
C_{3} & =\frac{15 G m_{1} m_{2} m_{3}\left(m_{1}-m_{2}\right)}{64\left(m_{1}+m_{2}\right)^{2} a_{2}\left(1-e_{2}^{2}\right)^{5 / 2}}\left(\frac{a_{1}}{a_{2}}\right)^{3},
\end{aligned}
$$

belong to the quadrupole and octupole terms in the interaction between the two binaries, respectively. All the remaining terms are due to GR effects: the precession of the inner periastron is taken into account in the evolution equation of $g_{1}$, whereas the back reaction of GW emission onto the inner binary is included in the evolution equations for $a_{1}, e_{1}$, and $H$. In particular, if $\mathrm{GW}$ emission is neglected, then $\mathrm{d} H / \mathrm{d} t=0$, as expected. We stress that such equations are obtained under an approximation that fails for $a_{2} \sim a_{1}$. This does not affect our results, as in this limit, the binaries are in the chaotic regime discussed in Section 2, and are therefore not evolved. Equations (10)-(17) present some interesting symmetries: apart from the trivial invariance for the exchange of the inner binary masses, $m_{1}^{\prime}=m_{2}$ and $m_{2}^{\prime}=m_{1}$, we notice also the invariance under the following transformation of the arguments of periastron: $g_{1}^{\prime}=g_{1}+\pi$ and $g_{2}^{\prime}=g_{2}+\pi$.

As a final note, in order to remove the divergence for $e_{1} \rightarrow$ 0 in the octupole term of equation (11), we solve the system of differential equations above in terms of the auxiliary variables $e_{1} \cos g_{1}, e_{1} \sin g_{1}, e_{2} \cos g_{2}$, and $e_{2} \sin g_{2}$, as suggested by Ford et al. (2000).

\section{ORBITAL EVOLUTION OF INNER COMPACT BINARIES}

The primary effect of the KL mechanism is the eccentricity growth that the inner binary can experience if certain conditions are satisfied. In the standard lore, the trigger conditions are derived with the assumptions that the total angular momentum is dominated by the outer binary and only the quadrupole order of approximation is considered. In this case, if the orbital planes of the inner and outer binary are misaligned, with relative inclination in the range $39^{\circ} \lesssim i \lesssim$ $141^{\circ}$ [see equation (6)], then secular exchanges of angular momentum between the two binaries can excite large oscillations of the relative inclination and of the inner eccentricity. When the initial relative inclination is close to $90^{\circ}$, the process shows its most extreme phenomenology: during the oscillations, the inner eccentricity can reach values close to unity that can potentially force the inner binary to coalesce ${ }^{6}$.

As pointed out in Section 2, this secular process can be suppressed if the orbit precesses (Holman et al. Ford et al. 2000; Miller \& Hamilton 2002; Blaes et al. 2002). Indeed, the resonance on which the KL mechanism relies strongly depends on the coherent piling up of the perturbation exerted by the

\footnotetext{
${ }^{6}$ More precisely, when a relevant fraction of the total angular momentum of the triplet is provided by the inner binary, the condition $e_{1} \rightarrow 1$ occurs at relative inclinations greater than $90^{\circ}$ (see, e.g. Lidov \& Ziglin 1976; Miller \& Hamilton 2002).
}

third body. If the inner binary starts to precess with a timescale much shorter than that of the KL oscillation, then the coherence is destroyed and the process is severely inhibited. For compact objects, the most relevant form of precession is the relativistic one. Therefore, in order not to overestimate the effect of the KL oscillation, the inclusion of this relativistic effect is crucial. In contrast, if the time-scale associated to the KL mechanism is shorter than that of the relativistic precession, then the process is only partially perturbed and a triple system can experience eccentricity excitations.

In Figures 3 and 4, we show two representative cases that describe the evolution of a BHNS and an NSNS binary, respectively, obtained by integrating equations (10)-(17). In both cases, the effect of secular evolution is clearly visible and drives the compact binary to coalescence within a time much shorter than the coalescence time for GW emission only. The upper and lower panels of the two figures show the evolution of the inner semi-major axis and of the inner eccentricity, respectively. The left panels describe the whole evolution of the inner compact binary up to coalescence. Note that single KL cycles cannot be resolved, as the oscillations proceed on a time-scale much shorter than that of the complete evolution. An interesting pattern is clearly visible in the evolution of the eccentricity: as the binary shrinks, the minimum inner eccentricity increases. As a consequence, the oscillation range of $e_{1}$ is reduced and the average value of $e_{1}$ experiences a net increment. This is due to the effect of GR corrections, which become stronger as the semi-major axis decreases and determine an increase of the minimum value of the relative inclination, which in turn increases the minimum eccentricity. This phenomenology persists until the semi-major axis has shrunk by nearly one order of magnitude. At that time, the KL mechanism is not efficient any longer in driving the dynamics of the systems. Then, the GW emission eventually takes over and quickly drives the binary towards coalescence. We mark this point with dashed vertical lines in the left and central panels of Figures 3 and 4, respectively. We computed it as the moment when the residual time to merger and the GW time-scale differ by less than $1 \%$. Due to the oscillatory behaviour of the eccentricity, for the evaluation of the GW time-scale [equation (1)], we employ orbital elements averaged over one quadrupole oscillation, i.e. $t_{\mathrm{GW}}=t_{\mathrm{GW}}\left(\langle a\rangle_{\mathrm{KL}}\right.$, $\left.\langle e\rangle_{\mathrm{KL}}\right)$.

Interesting patterns can be appreciated by zooming into different time-slices of the evolution, as represented in the central and right-hand panels. The central panels show a zoom-in on a time length comparable to the octupole timescale of the systems, whereas the right-hand panels focus on the quadrupole time-scale. When the eccentricity reaches the peak of the quadrupole oscillation with values close to unity (cf. the right-hand panels), the semi-major axis decreases sharply as a consequence of an efficient emission of GWs. Moreover, the octupole terms (cf. the central panels) clearly modulate the eccentricity growth and push its maximum value even further, determining a stronger and sharper extraction of orbital energy (cf. right-hand panels, 

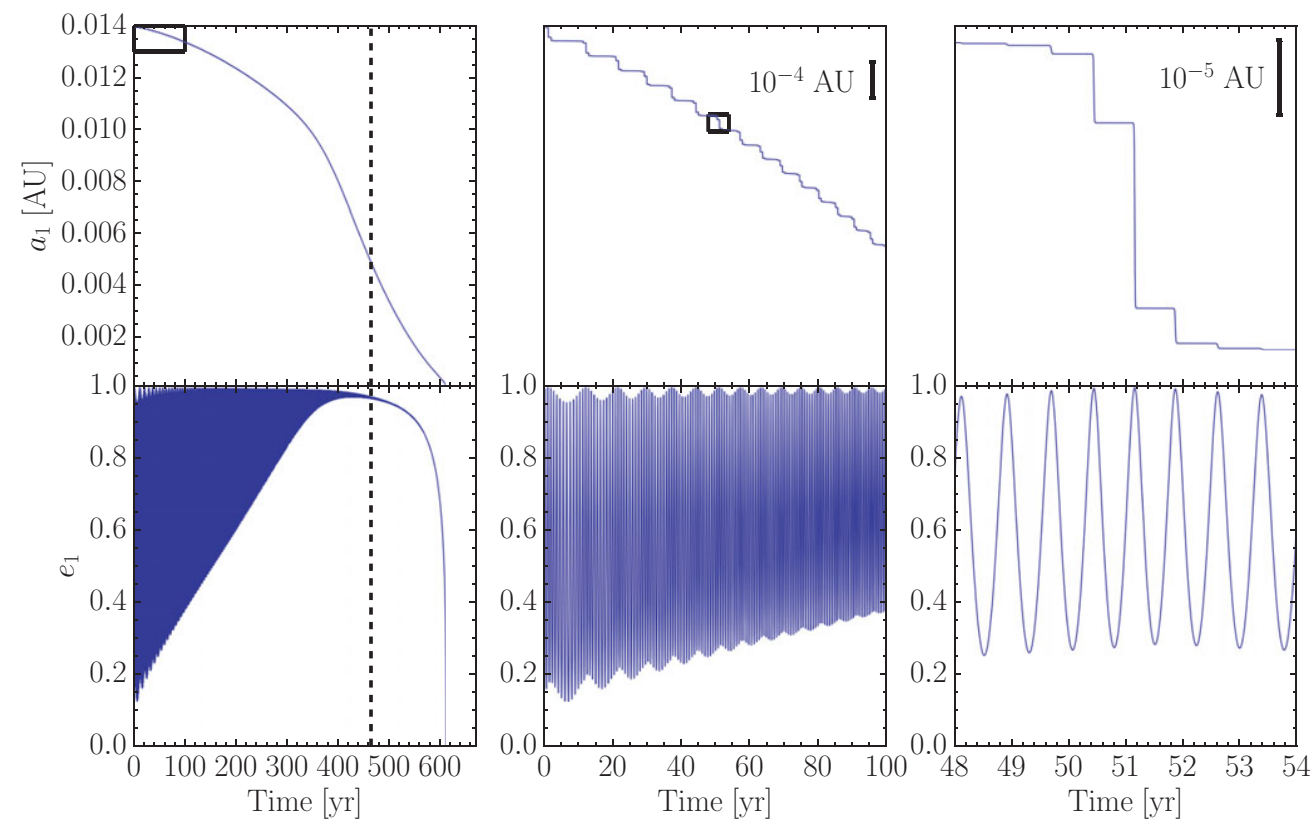

Figure 3. Triplet with a BHNS inner binary. The initial orbital parameters of the inner binary are $a_{1}=0.014 \mathrm{AU}, e_{1}$ $=0.150, m_{1}=9 \mathrm{M}_{\odot}, m_{2}=1.2 \mathrm{M}_{\odot}$, and $g_{1}=0^{\circ}$. The outer orbit is characterised by $a_{2}=0.306 \mathrm{AU}, e_{2}=0.6, g_{2}=$ $90^{\circ}, i=85^{\circ}$, and $m_{3}=16 \mathrm{M}_{\odot}$. Left panels: full evolution; Central panels: zoom-in on the octupole time-scale. Right panels: zoom-in on the quadrupole time-scale. Upper panels: evolution of the inner binary semi-major axis. Lower panels: evolution of the inner binary eccentricity. Note the sharp decrease of the semi-major axis when the eccentricity reaches its maximum value. The dashed vertical line corresponds to the point after which the KL mechanism does not significantly influence the evolution and GW emission takes over (see text for more details).

where a sharper decrease of $a_{1}$ is seen at the peak of the octupole modulation). Equations (7) and (9) provide analytical estimates of the quadrupole and octupole time-scales, respectively. The values provided by these expressions for the represented cases are $t_{\mathrm{KL} \text {, quad }} \sim 3.2(2.5) \mathrm{yr}$ and $t_{\mathrm{KL} \text {, oct }} \sim$ 25 (140) yr for the BHNS (NSNS) system. A comparison with the actual evolution reveals that the analytical estimates give values within a factor of a few compared with those inferred by the oscillations in Figures 3 and 4.

For both the simulated binaries, the octupole terms result to be quite relevant in the secular evolution, especially in the BHNS case. Indeed, a lower inner mass ratio $q$ enhances the strength of the octupole correction and reduce the associated oscillation time-scale, as it depends on the difference $m_{1}-$ $m_{2}$ [see, e.g. equations (9) and (19)]. Therefore, in addition to the reduced merger time-scale due to the higher mass with respect to the NSNS case, the lower mass ratio of the BHNS binary produces a much shorter octupole time-scale, which provides the possibility for the binary to reach a maximum in the eccentricity more frequently. Finally, the case of the NSNS binary, reported in Figure 4, also shows additional features during the evolution, in which after $t \sim 9.93 \times 10^{3} \mathrm{yr}$, a sharp change in the oscillation pattern is evident. This is due to the octupole terms that cause a switch from the libration regime (i.e. oscillation around $g_{1}=\pi / 2$ ) to the circulation regime (i.e. monotonic increase of $g_{1}$ in the range $[0,2 \pi]$ ) of the inner argument of pericentre (see discussion in Blaes et al. 2002), on a time-scale of a few times the octupole time- scale. In the latter regime, the minimum eccentricity is higher, which produces slightly more efficient $\mathrm{GW}$ emission.

Figures 3 and 4 show how the features of the KL mechanism change when mass and mass ratio of the inner binary vary. We take the converse approach in Appendix A, where we report a systematic exploration of the parameter space through a selected grid. We explore a few representative cases, both with NSNS and BHNS as inner binaries. We fix the masses of the inner component and vary all the other parameters that characterise the triplet. From our analysis, the most important parameters for the KL efficiency are the outer semi-major axis and the relative inclination. We address the interested reader to Appendix A for full details.

\section{COALESCENCE TIME-SCALE FOR STELLAR TRIPLET DISTRIBUTIONS}

To test the impact of triple system dynamics on the merger time-scale of a population of compact binaries, we generate different populations of triplets, all characterised by an inner compact binary and an orbiting outer star. We consider separately NSNS and BHNS inner binaries, and we vary the distribution of the inner semi-major axis between two cases, for a total of four different populations. The initial conditions characterising each triplet are generated through Monte Carlo sampling. A set of distributions is common to all populations and it includes the following: 

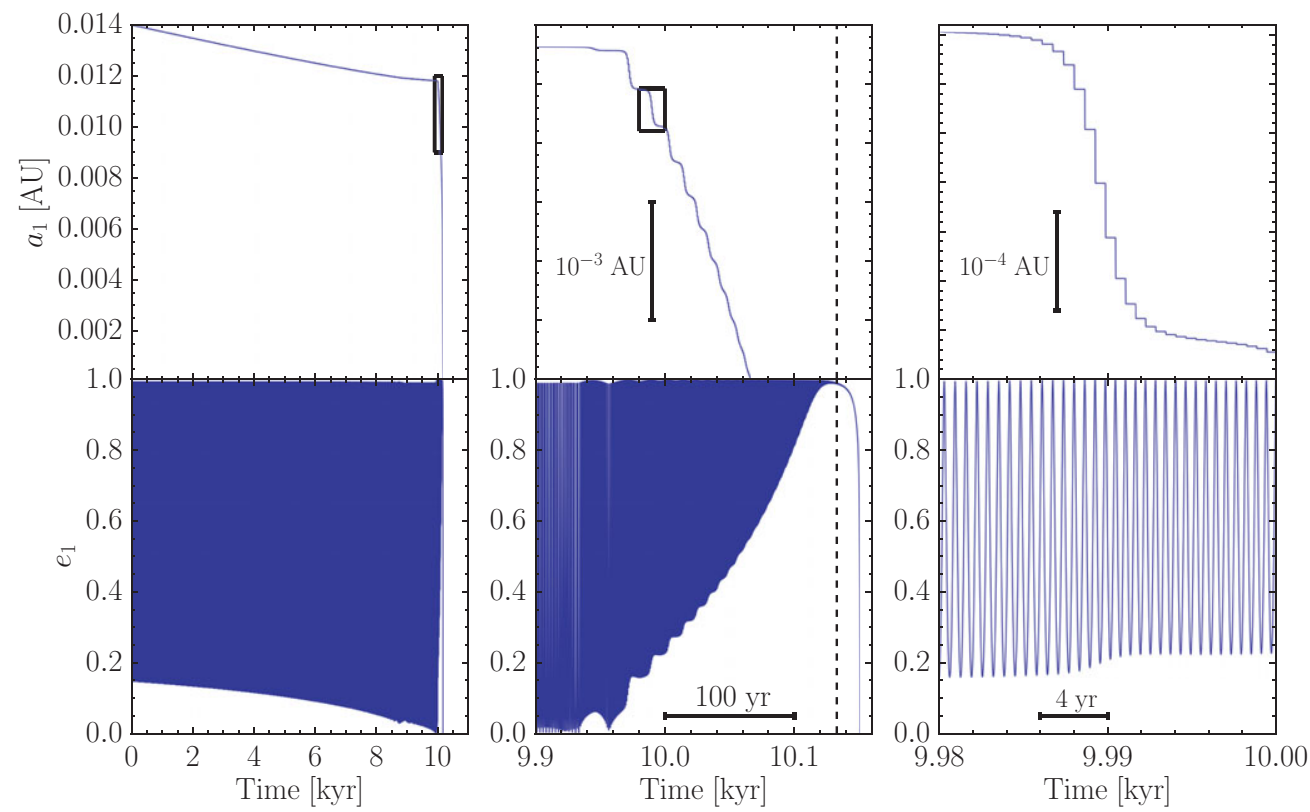

Figure 4. Same as Figure 3, except that the inner binary is an NSNS system with masses $\left(m_{1}, m_{2}\right)=(1.6,1.2) \mathrm{M}_{\odot}$ and $g_{1}=90^{\circ}$. Note the change of phenomenology around $t \sim 9.93 \times 10^{3} \mathrm{yr}$ when, because of the octupole term, the argument of pericentre of the inner binary changes from a libration to a circulation regime (see text).

- For $g_{1}$ and $g_{2}$, uniform distributions between 0 and $2 \pi$, and between 0 and $\pi$, respectively. The precise value of the two arguments of periastron depends on the details of the triplet formation. We assume isotropy and no correlation between the formation of the inner and outer binary. Moreover, we employ the symmetry presented at the end of Section 3 to halve the range of $g_{2}$.

- For $i$, a uniform distribution in $\cos i$ between -1 and 1 , which is equivalent to an isotropic probability for the direction of $\mathbf{G}_{2}$ with respect to $\mathbf{G}_{1}$.

- For $m_{3}$, Salpeter (1955) distribution with slope -2.3 between 3 and $15 \mathrm{M}_{\odot}$ (see the discussion of $m_{3}$ in Section 2).

- For $e_{1}$, a uniform distribution between 0 and 1 , because the observed NSNS binaries have a broad distribution and the actual value of $e_{1}$ does not have a strong impact on the evolution of the triplet.

- for $a_{2}$ and $e_{2}$, a linear distribution, i.e. $f(x) \propto x$, between $3 \times 10^{-2}$ and $10 \mathrm{AU}$, and between 0 and 1 , respectively. This kind of distribution is expected to be appropriate when triplets form dynamically (Heggie 1975).

For the NS masses in NSNS (BHNS) inner binaries, we consider $1.0 \leqslant m_{\mathrm{NS}} \leqslant 2.4 \mathrm{M}_{\odot}$ and we assume a Gaussian distribution centred around $1.4 \mathrm{M}_{\odot}\left(1.8 \mathrm{M}_{\odot}\right)$, with standard deviation $0.13 \mathrm{M}_{\odot}\left(0.18 \mathrm{M}_{\odot}\right)$ (Dominik et al. 2012). For the $\mathrm{BH}$ masses in BHNS inner binaries, we take $5 \leqslant m_{\mathrm{BH}} \leqslant 30 \mathrm{M}_{\odot}$, and we also assume a Gaussian distribution centred around 8 $\mathrm{M}_{\odot}$, with standard deviation $0.42 \mathrm{M}_{\odot}$ (Dominik et al. 2012). Finally, for the inner binary separation, we consider two possibilities: case $\mathrm{A}$, a distribution uniform in $\log _{10}\left(a_{1}\right)$; and case
$\mathrm{B}$, a distribution uniform in $a_{1}$. The orbital parameter distributions used to generate the triplets are summarised in the upper part of Table 2.

For each population, we randomly generate $N$ triple systems and we distinguish among precessing $(P)$, unstable $(U)$, and stable, non-precessing $(S)$ systems according to equations (4) and (8). Clearly, $N=P+U+S$. We produce $N$ triple systems such that $S=2000$. For the precessing systems, the coalescence time is assumed to be $t_{\mathrm{GW}}$, independent of the presence of the third external body. For unstable systems, we assume that the inner binary is always disrupted by the presence of the third body, which probably ejects the lighter compact object (i.e. the NS) from the innermost binary ${ }^{7}$. Thus, these systems will never lead to a compact binary coalescence when considered as part of a triple system. Finally, for the stable, non-precessing triples, we compute the merger time by integrating the equations of motion [cf. equations (10)-(17)]. We compare the distribution of the merger times for the triple systems with the distribution of $t_{\mathrm{GW}}$ for the $N$ inner binaries (i.e. always neglecting the effect of the third body). We normalise both distributions to $N$ to find the fraction of inner binaries that coalesce within $10^{8} \mathrm{yr}$, with and without the presence of the third body. In the lower part of Table 2, we summarise the results obtained for our four populations.

In Figure 5, we show our results for the NSNS distributions, both in the case of a uniform distribution in $a_{1}$ (left panel, case A) and in $\log _{10}\left(a_{1}\right)$ (right panel, case B). The

\footnotetext{
${ }^{7}$ We verified our assumption by simulating the triplet evolution of a large sub-sample of the unstable systems using the code developed in Bonetti et al. (2016).
} 
Table 2. Top: Summary of the distributions applied to produce the population of triple systems discussed in Section 5. Bottom: Summary of the results obtained from the above populations. $S, P$, and $U$ represent the number of stable non-processing, precessing, and unstable triple system in each population, respectively. $X_{\mathrm{GW}, 8}$ is the number of system of type $X$ whose inner binary has a GW-coalescence time-scale shorter than $10^{8} \mathrm{yr}$ without considering the third body perturbation, whereas $S_{\mathrm{M}, 8}$ is the number of triple stable, non-precessing systems whose merger time-scale is shorter than $10^{8} \mathrm{yr}$. The comparison between the last two rows shows the boosting effect of triple interactions.

\begin{tabular}{|c|c|c|c|c|}
\hline & NSNS, case A & NSNS, case B & BHNS, case A & BHNS, case B \\
\hline & \multicolumn{4}{|c|}{ Distributions } \\
\hline$g_{1}$ & \multicolumn{4}{|c|}{ Uniform in $[0,2 \pi]$} \\
\hline$g_{2}$ & \multicolumn{4}{|c|}{ Uniform in $[0, \pi]$} \\
\hline$m_{3}\left[\mathrm{M}_{\odot}\right]$ & \multicolumn{4}{|c|}{ Salpeter power law (slope -2.3 ), in $[3,15]$} \\
\hline$e_{1}$ & \multicolumn{4}{|c|}{ Uniform in $[0,1]$} \\
\hline$e_{2}$ & \multicolumn{4}{|c|}{ Linear in $[0,1]$} \\
\hline$a_{2}[\mathrm{AU}]$ & \multicolumn{4}{|c|}{ Linear in $[0.03,10]$} \\
\hline $\cos i$ & \multicolumn{4}{|c|}{ Uniform in $[-1,1]$} \\
\hline$\overline{m_{1}\left[\mathrm{M}_{\odot}\right]}$ & \multicolumn{2}{|c|}{$\begin{array}{l}\text { Gaussian in }[1.0,2.4] \\
\left\langle m_{1}\right\rangle=1.4, \sigma=0.13\end{array}$} & \multicolumn{2}{|c|}{$\begin{array}{l}\text { Gaussian in [5.0,30] } \\
\left\langle m_{1}\right\rangle=8, \sigma=0.42\end{array}$} \\
\hline$m_{2}\left[\mathrm{M}_{\odot}\right]$ & \multicolumn{2}{|c|}{ Gaussian in $[1.0,2.4]$} & \multicolumn{2}{|c|}{ Gaussian in $[1.0,2.4]$} \\
\hline \multirow[t]{2}{*}{$\overline{a_{1}[\mathrm{AU}]}$} & $\begin{array}{c}\text { Unif. in } \\
{[0.003,0.3]}\end{array}$ & $\begin{array}{c}\text { Unif. in } \\
\log _{10}[0.003,0.3]\end{array}$ & $\begin{array}{c}\text { Unif. in } \\
{[0.003,0.3]}\end{array}$ & $\begin{array}{c}\text { Unif. in } \\
\log _{10}[0.003,0.3]\end{array}$ \\
\hline & \multicolumn{4}{|c|}{ Results } \\
\hline $\bar{N}=S+P+U$ & 3346 & 3897 & 3297 & 5123 \\
\hline$S / N$ & 0.5977 & 0.5132 & 0.6066 & 0.3904 \\
\hline$S_{\mathrm{M}, 8} / N$ & 0.0607 & 0.0426 & 0.0874 & 0.0509 \\
\hline$S_{\mathrm{GW}, 8} / N$ & 0.0093 & 0.0159 & 0.0173 & 0.0189 \\
\hline$P / N$ & 0.0511 & 0.2969 & 0.1110 & 0.4540 \\
\hline$P_{\mathrm{GW}, 8} / N$ & 0.0254 & 0.1499 & 0.0658 & 0.3475 \\
\hline$\overline{U / N}$ & 0.3512 & 0.1899 & 0.2824 & 0.1556 \\
\hline$U_{\mathrm{GW}, 8} / N$ & 0.0036 & 0.0100 & 0.0103 & 0.0197 \\
\hline$\left(S_{\mathrm{M}, 8}+P_{\mathrm{GW}, 8}\right) / N$ & 0.0861 & 0.1925 & 0.1532 & 0.3984 \\
\hline$(S+P+U)_{\mathrm{GW}, 8} / N$ & 0.0383 & 0.1758 & 0.0934 & 0.3861 \\
\hline
\end{tabular}

precessing triplets merging within $t_{\text {merge }}$ are common both to the triple and binary distributions (green star bars). The KL mechanism leads to an increase of the merger rate (red empty bars), even when considering the systematic disruption of the inner binary when part of unstable triple systems. In case $\mathrm{A}$, the uniform distribution of the inner semi-major axis, combined with the linear distribution of the outer semimajor axis, favours the presence of stable, non-precessing triplets ( $\sim 60 \%$ of the cases). The few precessing systems are characterised by tight inner binaries, which coalesce within $10^{8} \mathrm{yr}$ in $\sim 50 \%$ of the cases. The remaining unstable systems have rather large initial $a_{1}$ and only a very small fraction of their inner compact binaries $(\sim 1 \%)$ would merge as isolated binaries. Overall, only $3.8 \%$ of the inner systems of this population would coalesce within $10^{8} \mathrm{yr}$ as isolated binaries. For stable, non-precessing systems, the KL mechanism causes a fast merger of the inner binary in one case out of ten, which is increased by a factor of 6.5 compared with the fraction of merging isolated binaries. Considering the whole population, the number of systems coalescing within $10^{8} \mathrm{yr}$ as triplets has increased by a factor 2.25 , to $8.6 \%$ of the population.
The $\log _{10}$-uniform distribution of inner semi-major axis used in case B produces qualitatively different results. The presence of a much larger number of tight inner binaries increases the number of precessing systems at the expense of the unstable and, less severely, of the stable, non-precessing systems. Also, in this case, more than $50 \%$ of the inner binaries contained inside the precessing triplets will coalesce anyway within $10^{8} \mathrm{yr}$. The KL mechanism increases the number of fast coalescences in stable, non-precessing systems by a factor of 2.7. However, due to the dominant presence of tight, precessing systems, the total fraction of fast coalescing systems increases only from 17.6 to $19.25 \%$, when passing from isolated binaries to triplets. The temporal distributions reported in Figure 5 suggest also that the number of coalescing systems increases with $t_{\text {merge }}$ for all system types. However, the increase is more pronounced for precessing and unstable systems. Thus, the KL mechanism is very efficient in increasing the number of mergers on extremely short time-scales $\left(t_{\text {merge }}<10^{5} \mathrm{yr}\right)$.

The results obtained for the BHNS inner binary cases are reported in Figure 6, both for a uniform distribution in $a_{1}$ (left panel, case A) and in $\log _{10}\left(a_{1}\right)$ (right panel, case B). The 

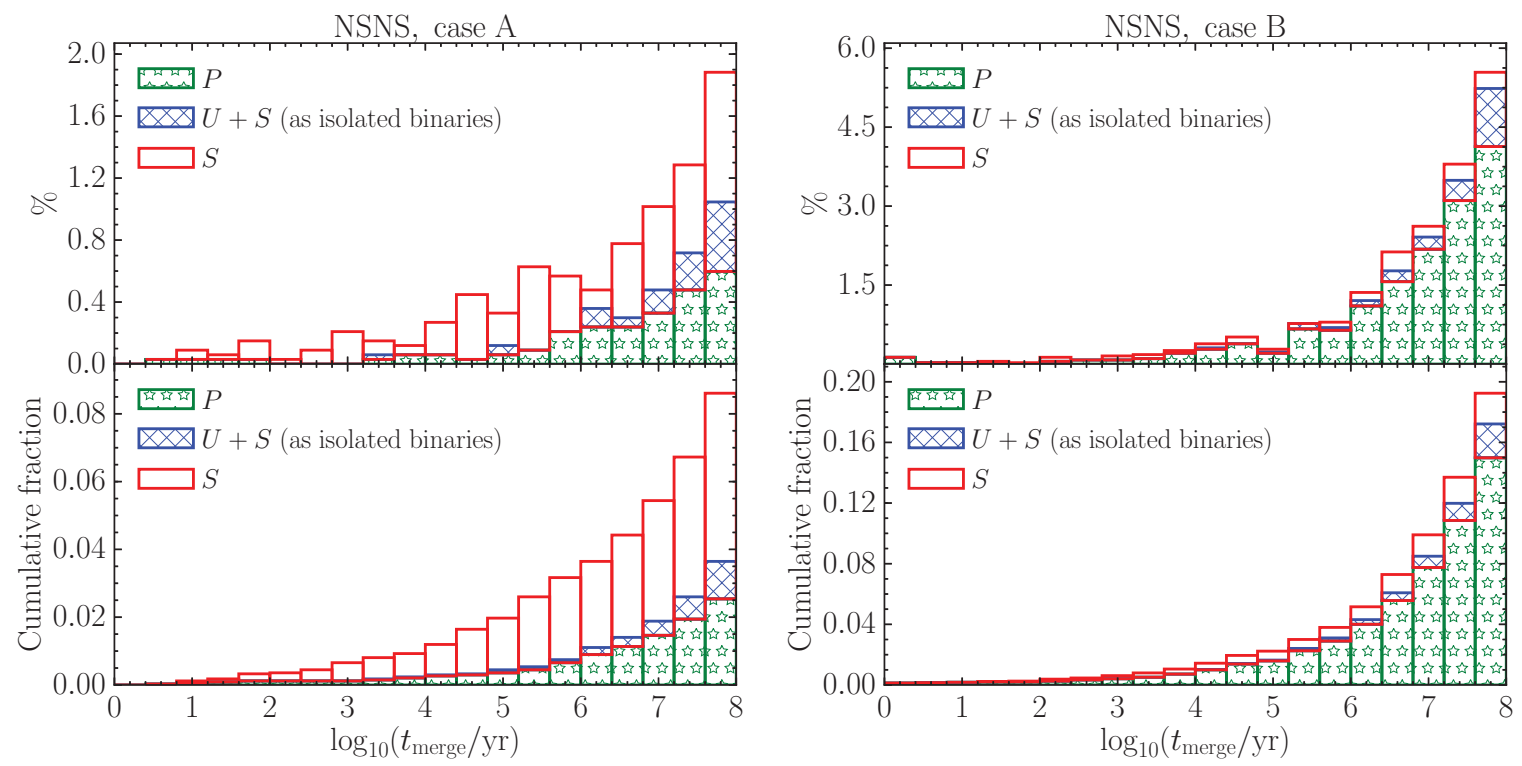

Figure 5. Comparison of the distributions of the merger time-scale below $10^{8} \mathrm{yr}$ for NSNS binaries in triplets $\left(t_{\mathrm{merge}}\right)$ and for the same binaries assumed as isolated (i.e. $t_{\mathrm{GW}}$ ). Details of the distributions are specified in Table 2. Green bars (filled with stars) include triplets for which the relativistic precession of the inner binary strongly inhibits the effect of secular effects. For these systems, we assume $t_{\text {merge }}$ $\approx t_{\mathrm{GW}}$. Blue bars (filled with lines) include $t_{\mathrm{GW}}$ of the inner binary both for hierarchical, non-precessing triplets and unstable triplets. Red bars (unfilled) contain hierarchical, non-precessing systems considered as triplets. Left panels: initial inner binary distribution uniform in $a_{1}$. Right panels: initial inner binary distribution uniform in $\log _{10}\left(a_{1}\right)$. Upper panels: percentage of runs. Lower panels: cumulative fraction of runs.
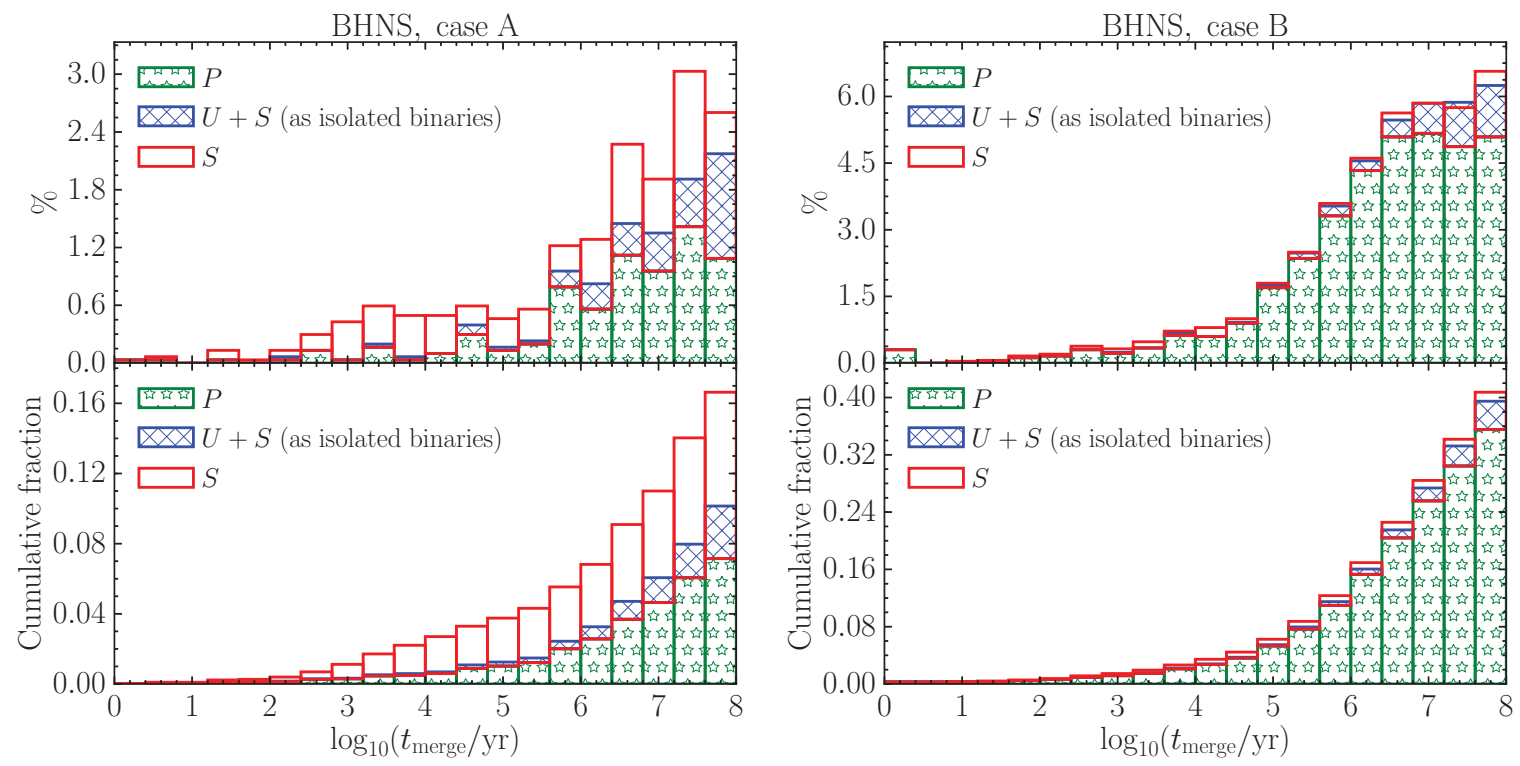

Figure 6. Same as Figure 5, but for BHNS inner binaries.

qualitative behaviour of the NSNS populations described above is also valid in the case of BHNS populations. The presence of a stellar-mass $\mathrm{BH}$ in the inner binary increases $m_{1}+m_{2}$, leading to a more efficient $\mathrm{GW}$ emission and a significantly shorter $t_{\mathrm{GW}}$, since $t_{\mathrm{GW}} \propto\left[\left(m_{1}+m_{2}\right) m_{1} m_{2}\right]^{-1}$ [see equation (1)]. It also increases the stability of triple systems [see equation (4)], but favours the relativistic precession of the inner binary [see equation (8)]. Moreover, the combina- tion with the $a_{1}^{4 / 3}$ dependence in equation (8) makes the occurrence of precession even more pronounced, moving from case A to case B. The more massive inner binary makes the KL resonance induced by the third body less efficient [this is visible, for example, on the longer time-scale for the dominant quadrupole oscillations; see equation (7)]. On the other hand, the larger mass difference potentially increases the importance of octupole modulation (see Section 4). For a 
uniform distribution in $a_{1}$ (case $\mathrm{A}$ ), the largest contribution to the number of inner binaries that would coalesce as isolated binaries is provided by tight precessing systems $(6.58 \%$ of the whole population). The KL mechanism increases the number of compact binaries that have a fast coalescence in stable, non-precessing systems by a factor of 5, and up to $8.74 \%$ of the population, i.e. in a way similar to what reported for the NSNS population of case A. In total, the fraction of BHNS binaries that coalesce within $10^{8} \mathrm{yr}$ has increased from $9.34 \%$ as isolated binaries to $15.3 \%$ as inner binaries of a population of triplets. The larger absolute values, compared with the NSNS population, are simply due to the more efficient GW emission, while the impact of the KL mechanism has slightly decreased, due to the more massive inner binary. The even more reduced impact of the KL mechanism on the fraction of the fast coalescing, stable, non-precessing systems becomes marginal in case B of the BHNS population. For the latter, the largest fraction ( $\gtrsim 38 \%$ ) of fast coalescing system is represented by precessing systems, which merge within $10^{8} \mathrm{yr}$ in $\sim 75 \%$ of the cases.

\section{DISCUSSION AND CONCLUSIONS}

In this work, we have analysed the impact of the KL mechanism on the merger rate of compact binaries (both BHNS and NSNS) in the early stage of the cosmological evolution. Our investigations are motivated by the observation of $r$-process elements in old, metal-poor stars, which demands the occurrence of $r$-process nucleosynthesis for $[\mathrm{Fe} / \mathrm{H}]<-3$ (corresponding to a delay of $\sim 10^{8} \mathrm{yr}$ after the birth of the first stars in the case of efficient elemental mixing in the galactic interstellar medium). We have verified that the KL mechanism can, under certain conditions, be important in shaping the merger rate of compact binaries. Our results confirm previous findings of Thompson (2011), who showed that the $\mathrm{KL}$ mechanism can be relevant in increasing the merger rate of compact binaries on time-scales comparable to the Hubble time. However, we have specialised to the case of fast $\left(\sim 10^{8} \mathrm{yr}\right)$ mergers, for which we have found the following.

On the one hand, if the main compact binary formation channel favours the occurrence of tight compact systems (for instance, with $a_{1}$ distributed uniformly in logarithm), then the influence of the KL mechanism is negligible because the merger fraction increases by only a few percent. This is due to the stronger relativistic precession that characterises tighter binaries and destroys the KL resonance. However, in this scenario, given the smaller average inner separations, a significant fraction of binaries efficiently merges in short timescales without any external influence (see, e.g. Beniamini, Hotokezaka, \& Piran 2016).

On the other hand, if the distribution of the semi-major axes favour the formation of wider inner compact binaries, then the merger rate of NSNS and BHNS binaries can be increased up to a factor of two because of secular triple interactions. Since in this situation the fraction of tight binaries that efficiently merge in less than $100 \mathrm{Myr}$ is low (only a few percent), triple interactions should not be neglected and the KL mechanism can be crucial, if CBMs are the main site for the production of $r$-process elements in the early Universe.

A remarkable feature of the enhanced CBM rate due to the $\mathrm{KL}$ mechanism is the occurrence of ultra-fast merger events ( $\lesssim 10 \mathrm{Myr}$ ). Such a reduced merger time-scale could be crucial to explain the observed abundances in $r$-process enriched ultra-faint dwarf galaxies (e.g. Reticulum II) with a single CBM event (Safarzadeh \& Scannapieco 2017). Indeed, the shallow potential well of the ultra-faint dwarf halos, combined with the potentially large natal kick of compact binaries, requires ultra-fast mergers so that the merger does not happen outside the galaxy and to prevent interstellar medium enrichment (see, e.g. Safarzadeh \& Côté 2017, but see also Beniamini et al. 2016, for the possible impact of low natalkick, tight binaries).

We have performed our study under the assumption of secular evolution, up to octupole-order KL equations. However, we cannot exclude that the inclusion of higher order effects or the study of non-hierarchical situations could be relevant, at least for a part of the wide parameter space. A more detailed study, employing direct integration schemes, will be the subject of forthcoming investigations.

Despite the potential relevance of the KL mechanism for the merger rate of compact binaries, several questions concerning the formation rate and properties of triple systems remain unanswered. A first question is whether hierarchical triple systems can easily form and if they are frequent enough. The total fraction of massive stars that are located in multiple systems is $\gtrsim 80 \%$ (Duchêne \& Kraus 2013), with a significant portion $(\sim 10 \%)$ in triple or even quadruple systems (see Belczynski et al. 2014, and references therein). Recent hydrodynamical simulations of primordial star formation predict that the collapse of metal-free clouds of $\mathrm{H}$ and He likely forms multiple systems (Stacy, Greif, \& Bromm 2010; Clark et al. 2011; Girichidis et al. 2012). Moreover, the initial mass function for metal-free stars can differ significantly from what we observe at later epochs (e.g. Hartwig et al. 2015, and references therein) and increase the presence of more stable high-mass tertiary components, for which we expect the KL mechanism to be more efficient. A second question concerns the places and the channels through which these systems can be born. Triple systems can form either in GCs or in the GF. The formation probability is larger in GCs, because they are denser stellar environments. Indeed, the formation of compact binaries in high-redshift GCs can already enhance the merger rate in the early Universe (Ramirez-Ruiz et al. 2015). However, in a Milky Way-like galaxy, only $\sim 10^{7}$ out of $\sim 10^{11}$ stars are located in GCs. Thus, triple systems in the GF are also relevant. A first channel to produce hierarchical triple systems is in-situ formation. This can happen both in GCs and in the GF. For fixed energy and angular momentum, there is more phase space in which the lighter object is outside. In this case, the inner system can evolve in a compact binary, while the outer body stays an ordinary star. Although the inner and outer angular momenta are initially 
aligned, asphericity in the SN explosions of the inner binary can lead more easily to misaligned configurations. Another channel is the dynamical formation of a triple system from the capture of a third body by a compact binary. However, because in the Newtonian point-mass approximation, the orbits are time-reversible, the formation of a stable hierarchical triple is only possible if energy can be dissipated, e.g. via tidal effects or the emission of GWs (see Bailyn 1989). Finally, another feasible channel is the interaction between a compact binary and another wider binary, which can trigger the ejection of the lighter component of the latter and the formation of a stable triplet. Dynamical channels are expected to be more likely in GCs where perturbations due to the global distribution of stars are expected to be more relevant for wider, triple systems than for binaries. If these perturbations induce changes in the relative inclination, the probability to access the KL-favourable range could be increased (see, e.g. VanLandingham et al. 2016). If they trigger instabilities or exchanges, this could lead to a shrinking of the semi-major axis or to an increase of the eccentricity of the semi-major axis.

\section{ACKNOWLEDGEMENTS}

The authors thank A. Arcones, M. Pignatari, M. Safarzadeh, F.-K. Thielemann, and B. Wehmeyer for useful discussions. MB and MD acknowledge the CINECA award under the ISCRA initiative, for the availability of high-performance computing resources and support. AP acknowledges support from the Helmholtz-University Investigator grant no. VH-NG-825, and from the INFN project 'High Performance data Network' funded by CIPE. This work was supported by a grant from the Swiss National Supercomputing Centre (CSCS) under project ID 667. AP thanks also the GSI Helmholtzzentrum für Schwerionenforschung $\mathrm{GmbH}$ for the usage of computational resources. PRC acknowledges support by the Tomalla foundation.

\section{REFERENCES}

Abbott, B. P., et al. 2017a, PhRvL, 119, 161101

Abbott, B. P., et al. 2017b, ApJ, 848, L12

Antognini, J. M. O., \& Thompson, T. A. 2016, MNRAS, 456, 4219

Antonini, F., Chatterjee, S., Rodriguez, C. L., Morscher, M., Pattabiraman, B., Kalogera, V., \& Rasio, F. A. 2016, ApJ, 816, 65

Antonini, F., \& Perets, H. B. 2012, ApJ, 757, 27

Arcones, A., \& Thielemann, F.-K. 2013, JPhG, 40, 013201

Argast, D., Samland, M., Thielemann, F.-K., \& Qian, Y.-Z. 2004, A\&A, 416, 997

Arlandini, C., Käppeler, F., Wisshak, K., Gallino, R., Lugaro, M., Busso, M., \& Straniero, O. 1999, ApJ, 525, 886

Bailyn, C. D. 1989, ApJ, 341, 175

Bauswein, A., Goriely, S., \& Janka, H.-T. 2013, ApJ, 773, 78

Belczynski, K., Buonanno, A., Cantiello, M., Fryer, C. L., Holz, D. E., Mandel, I., Miller, M. C., \& Walczak, M. 2014, ApJ, 789, 120

Beniamini, P., Hotokezaka, K., \& Piran, T. 2016, ApJ, 829, L13

Blaes, O., Lee, M. H., \& Socrates, A. 2002, ApJ, 578, 775

Bonetti, M., Haardt, F., Sesana, A., \& Barausse, E. 2016, MNRAS, 461,4419
Bovard, L., Martin, D., Guercilena, F., Arcones, A., Rezzolla, L., \& Korobkin, O. 2017, PhRv D, 96, 124005

Bromm, V., Coppi, P. S., \& Larson, R. B. 2002, ApJ, 564, 23

Cescutti, G., Romano, D., Matteucci, F., Chiappini, C., \& Hirschi, R. 2015, A\&A, 577, A139

Chornock, R., et al. 2017, ApJ, 848, 19

Clark, P. C., Glover, S. C. O., Smith, R. J., Greif, T. H., Klessen, R. S., \& Bromm, V. 2011, Science, 331, 1040

Coulter, D. A., et al. 2017, Science, 358, 1556

Dominik, M., Belczynski, K., Fryer, C., Holz, D. E., Berti, E., Bulik, T., Mandel, I., \& O’Shaughnessy, R. 2012, ApJ, 759, 52

Duchêne, G., \& Kraus, A. 2013, ARA\&A, 51, 269

Eichler, D., Livio, M., Piran, T., \& Schramm, D. N. 1989, Nature, 340, 126

Fernández, R., \& Metzger, B. D. 2013, MNRAS, 435, 502

Fernández, R., \& Metzger, B. D. 2016, ARNPS, 66, 23

Ford, E. B., Kozinsky, B., \& Rasio, F. A. 2000, ApJ, 535, 385

Foucart, F., et al. 2015, PhRvD, 91, 124021

Freiburghaus, C., Rosswog, S., \& Thielemann, F.-K. 1999, ApJ, $525, \mathrm{~L} 121$

Fujimoto, S.-I., Nishimura, N., \& Hashimoto, M.-A. 2008, ApJ, 680, 1350

Girichidis, P., Federrath, C., Banerjee, R., \& Klessen, R. S. 2012, MNRAS, 420, 613

Hartwig, T., Bromm, V., Klessen, R. S., \& Glover, S. C. O. 2015, MNRAS, 447, 3892

Heggie, D. C. 1975, MNRAS, 173, 729

Hirai, Y., Ishimaru, Y., Saitoh, T. R., Fujii, M. S., Hidaka, J., \& Kajino, T. 2015, ApJ, 814, 41

Hoffman, L., \& Loeb, A. 2007, MNRAS, 377, 957

Hoffman, R. D., Woosley, S. E., \& Qian, Y.-Z. 1997, NuPhA, 621, 397

Holman, M., Touma, J., \& Tremaine, S. 1997, Nature, 386, 254

Honda, S., Aoki, W., Ishimaru, Y., Wanajo, S., \& Ryan, S. G. 2006, ApJ, 643, 1180

Hotokezaka, K., Kiuchi, K., Kyutoku, K., Muranushi, T., Sekiguchi, Y., Shibata, M., \& Taniguchi, K. 2013, PhRvD, 88, 044026

Hotokezaka, K., Piran, T., \& Paul, M. 2015, NatPh, 11, 1042

Iwasawa, M., Funato, Y., \& Makino, J. 2006, ApJ, 651, 1059

Ji, A. P., Frebel, A., Chiti, A., \& Simon, J. D. 2016, Nature, 531, 610

Just, O., Bauswein, A., Pulpillo, R. A., Goriely, S., \& Janka, H.-T. 2015, MNRAS, 448, 541

Käppeler, F., Gallino, R., Bisterzo, S., \& Aoki, W. 2011, RvMP, 83, 157

Karakas, A. I., \& Lattanzio, J. C. 2014, PASA, 31, e030

Katz, B., Dong, S., \& Malhotra, R. 2011, PhRvL, 107, 181101

Korobkin, O., Rosswog, S., Arcones, A., \& Winteler, C. 2012, MNRAS, 426, 1940

Kozai, Y. 1962, AJ, 67, 591

Kulkarni, G., \& Loeb, A. 2012, MNRAS, 422, 1306

Lattimer, J. M., \& Schramm, D. N. 1974, ApJ, 192, L145

Lidov, M. L. 1962, P\&SS, 9, 719

Lidov, M. L., \& Ziglin, S. L. 1976, CeMec, 13, 471

Mardling, R. A., \& Aarseth, S. J. 2001, MNRAS, 321, 398

Martin, D., Perego, A., Arcones, A., Thielemann, F.-K., Korobkin, O., \& Rosswog, S. 2015, ApJ, 813, 2

Martin, D., Perego, A., Kastaun, W., \& Arcones, A. 2017, Class. Quantum Gravity, 35, 034001

Metzger, B. D. 2017, LRR, 20, 3

Miller, M. C., \& Hamilton, D. P. 2002, ApJ, 576, 894 
Mösta, P., et al. 2014, ApJ, 785, L29

Naoz, S., Farr, W. M., \& Rasio, F. A. 2012, ApJ, 754, L36

Naoz, S., Farr, W. M., Lithwick, Y., Rasio, F. A., \& Teyssandier, J. 2013, MNRAS, 431, 2155

Nicholl, M., et al. 2017, ApJ, 848, L18

Nishimura, N., Takiwaki, T., \& Thielemann, F.-K. 2015, ApJ, 810, 109

Perego, A., Rosswog, S., Cabezón, R. M., Korobkin, O., Käppeli, R., Arcones, A., \& Liebendörfer, M. 2014, MNRAS, 443, 3134

Peters, P. C. 1964, PhRv, 136, 1224

Pian, E., et al. 2017, Nature, 551, 67

Radice, D., Galeazzi, F., Lippuner, J., Roberts, L. F., Ott, C. D., \& Rezzolla, L. 2016, MNRAS, 460, 3255

Ramirez-Ruiz, E., Trenti, M., MacLeod, M., Roberts, L. F., Lee, W. H., \& Saladino-Rosas, M. I. 2015, ApJ, 802, L22

Roberts, L. F., et al. 2017, MNRAS, 464, 3907

Roederer, I. U., Cowan, J. J., Preston, G. W., Shectman, S. A., Sneden, C., \& Thompson, I. B. 2014, MNRAS, 445, 2970

Rosswog, S. 2015, IJMPA, 24, 30012

Safarzadeh, M., \& Côté, B. 2017, MNRAS, 471, 4488

Safarzadeh, M., \& Scannapieco, E. 2017, MNRAS, 471, 2088

Salpeter, E. E. 1955, ApJ, 121, 161

Shen, S., Cooke, R. J., Ramirez-Ruiz, E., Madau, P., Mayer, L., \& Guedes, J. 2015, ApJ, 807, 115

Sneden, C., Cowan, J. J., \& Gallino, R. 2008, ARA\&A, 46, 241

Sneden, C., et al. 2003, ApJ, 591, 936

Stacy, A., Greif, T. H., \& Bromm, V. 2010, MNRAS, 403, 45

Surman, R., McLaughlin, G. C., Ruffert, M., Janka, H.-T., \& Hix, W. R. 2008, ApJ, 679, L117

Symbalisty, E., \& Schramm, D. N. 1982, ApJ, 22, 143

Tanvir, N. R., et al. 2017, ApJ, 848, L27

Tauris, T. M., et al. 2017, ApJ, 846, 170

Thielemann, F.-K., Eichler, M., Panov, I. V., \& Wehmeyer, B. 2017, ARNPS, 67, 253

Thompson, T. A. 2011, ApJ, 741, 82

van de Voort, F., Quataert, E., Hopkins, P. F., Kereš, D., \& FaucherGiguère, C.-A. 2015, MNRAS, 447, 140

VanLandingham, J. H., Miller, M. C., Hamilton, D. P., \& Richardson, D. C. 2016, ApJ, 828, 77

Wanajo, S., Sekiguchi, Y., Nishimura, N., Kiuchi, K., Kyutoku, K., \& Shibata, M. 2014, ApJ, 789, L39

Wehmeyer, B., Pignatari, M., \& Thielemann, F.-K. 2015, MNRAS, 452, 1970

Winteler, C., Käppeli, R., Perego, A., Arcones, A., Vasset, N., Nishimura, N., Liebendörfer, M., \& Thielemann, F.-K. 2012, ApJ, 750, L22

Woosley, S. E., \& Heger, A. 2006, ApJ, 637, 914

Wu, M.-R., Fernández, R., Martínez-Pinedo, G., \& Metzger, B. D. 2016, MNRAS, 463, 2323

\section{A EXTENSIVE PARAMETER EXPLORATION}

In this appendix, we report on a broader parameter space exploration of hierarchical, non-precessing triple systems with few selected masses for the inner compact binary. The main goal of this study is to highlight which parameters are most relevant in shaping the KL efficiency, eventually causing binary coalescence. In Table 3, we summarise the surveyed parameter space and its sampling. For the NSNS (BHNS) case, we choose two (three) different mass combinations, and for each of them two further choices of the initial inner and outer arguments of pericentre (i.e. $g_{1}, g_{2}$ ). For $m_{3}$, we choose six values in the range $[1,16] \mathrm{M}_{\odot}$, whereas for the inner $\left(e_{1}\right)$ and outer $\left(e_{2}\right)$ eccentricities, we select six and four values uniformly spaced in the range [0,1], respectively. The inner $\left(a_{1}\right)$ and outer $\left(a_{2}\right)$ semi-major axes take instead five and six logarithmically spaced values from 0.005 to $0.3 \mathrm{AU}$ and from 0.03 to $10 \mathrm{AU}$, respectively. Finally, we choose the relative inclination uniformly spaced in the cosine from $30^{\circ}$ to $85^{\circ}$. In addition, according to the findings of Miller \& Hamilton (2002), we also choose to explore a single retrograde case with relative inclination of $95^{\circ}$.

In Figures 7-9, we report the merger fraction (colour-coded) of three representative cases [i.e. NSNS II and BHNS III with $\left(g_{1}, g_{2}\right)$ $=\left(180^{\circ}, 0^{\circ}\right)$, and BHNS II with $\left(g_{1}, g_{2}\right)=\left(90^{\circ}, 270^{\circ}\right)$; see Table 3] as a function of any possible combination $\left(p_{1}, p_{2}\right)$ of two different grid parameters. For every possible pair of values of $p_{1}$ and $p_{2}$, we consider the sample represented by stable and non-precessing triplets for which $t_{\mathrm{GW}}>10^{8} \mathrm{yr}$. The merger fraction is computed as the number of grid points for which $t_{\text {merge }}<10^{8} \mathrm{yr}$, normalised to the total number of points in the sample. ${ }^{8}$ A merger fraction close to one implies that the KL mechanism makes the (otherwise, slowly merging) inner binary always coalesce within $10^{8} \mathrm{yr}$, irrespective of all the other parameters. A merger fraction close to zero could correspond to a configuration of $p_{1}$ and $p_{2}$ for which the KL mechanism is not efficient enough, or for which stable, non-precessing systems are absent, or for which the inner binary coalesces within $10^{8} \mathrm{yr}$ even in the absence of triple interactions. As can be inferred from the plots, the parameter $a_{2}$ is the most relevant in shaping the merger fraction. Indeed, all combinations including $a_{2}$ show a strongly clustered pattern. The strong dependence on $a_{2}$ arises because the KL time-scales themselves depend on a high power of the outer semimajor axis [see equations (7) and (9)]. Therefore, mild variations in $a_{2}$ lead to large changes in the KL oscillation time-scale, which in turn control how frequently the maximum inner eccentricity is reached, with its resulting copious emission of GWs. A further important role is played by the relative inclination, which leads to a high merger fraction when its value is close to $90^{\circ}$. In contrast, although the tertiary mass, $m_{3}$, can affect the oscillation time-scale, it does not seem to have a critical impact in the explored mass range. These features are common both to NSNS and BHNS systems.

A further parameter which one might expect to be important is the inner semi-major axis, $a_{1}$, which strongly characterises the merger time-scale of compact binaries. However, it affects the merger fraction of binaries in triple systems only marginally. The reason has to be ascribed to our exploration strategy, which here is solely directed to the assessment of the KL efficiency and not to the overall merger fraction. Indeed, a large fraction of tight inner binaries precess [see equation (8)], or merge rapidly [see equation (1)], whereas wide inner binaries are more unstable [see equation (4)]. This explains the mild dependence on $a_{1}$ and also the sharp decreases (dark blue areas) that affect the merger fraction. The lower merger fractions visible for the BHNS cases are due to the more efficient GW emission, which increases significantly the number of binaries that would fast coalesce also as isolated binary.

\footnotetext{
${ }^{8}$ We assign a merger fraction of zero also in the case when there are no stable and non-precessing triplets for a specific combination of values of $p_{1}$ and $p_{2}$.
} 
Table 3. Parameter space sampling.

\begin{tabular}{|c|c|c|c|c|c|}
\hline \multicolumn{6}{|c|}{ Parameter space } \\
\hline & NSNS, I & NSNS, II & BHNS, I & BHNS, II & BHNS, III \\
\hline$m_{1}\left[\mathrm{M}_{\odot}\right]$ & 1.3 & 1.6 & 7.5 & 9.0 & 15 \\
\hline$m_{2}\left[\mathrm{M}_{\odot}\right]$ & 1.1 & 1.2 & 1.2 & 1.8 & 1.8 \\
\hline$\left(g_{1}, g_{2}\right)\left[^{\circ}\right]$ & \multicolumn{5}{|c|}{$\left(90^{\circ}, 270^{\circ}\right),\left(180^{\circ}, 0^{\circ}\right)$} \\
\hline$m_{3}\left[\mathrm{M}_{\odot}\right]$ & \multicolumn{5}{|c|}{$1,4,7,10,13,16$} \\
\hline$e_{1}$ & \multicolumn{5}{|c|}{$0.15,0.3,0.45,0.6,0.75,0.9$} \\
\hline$a_{1}[\mathrm{AU}]$ & \multicolumn{5}{|c|}{$0.005,0.014,0.039,0.108,0.3$} \\
\hline$e_{2}$ & \multicolumn{5}{|c|}{$0.2,0.4,0.6,0.8$} \\
\hline$a_{2}[\mathrm{AU}]$ & \multicolumn{5}{|c|}{$0.03,0.096,0.306,0.979,3.129,10$} \\
\hline $\cos i$ & \multicolumn{5}{|c|}{$0.866,0.779,0.693,0.606,0.52,0.433,0.347,0.26,0.174,0.087,-0.087$} \\
\hline
\end{tabular}

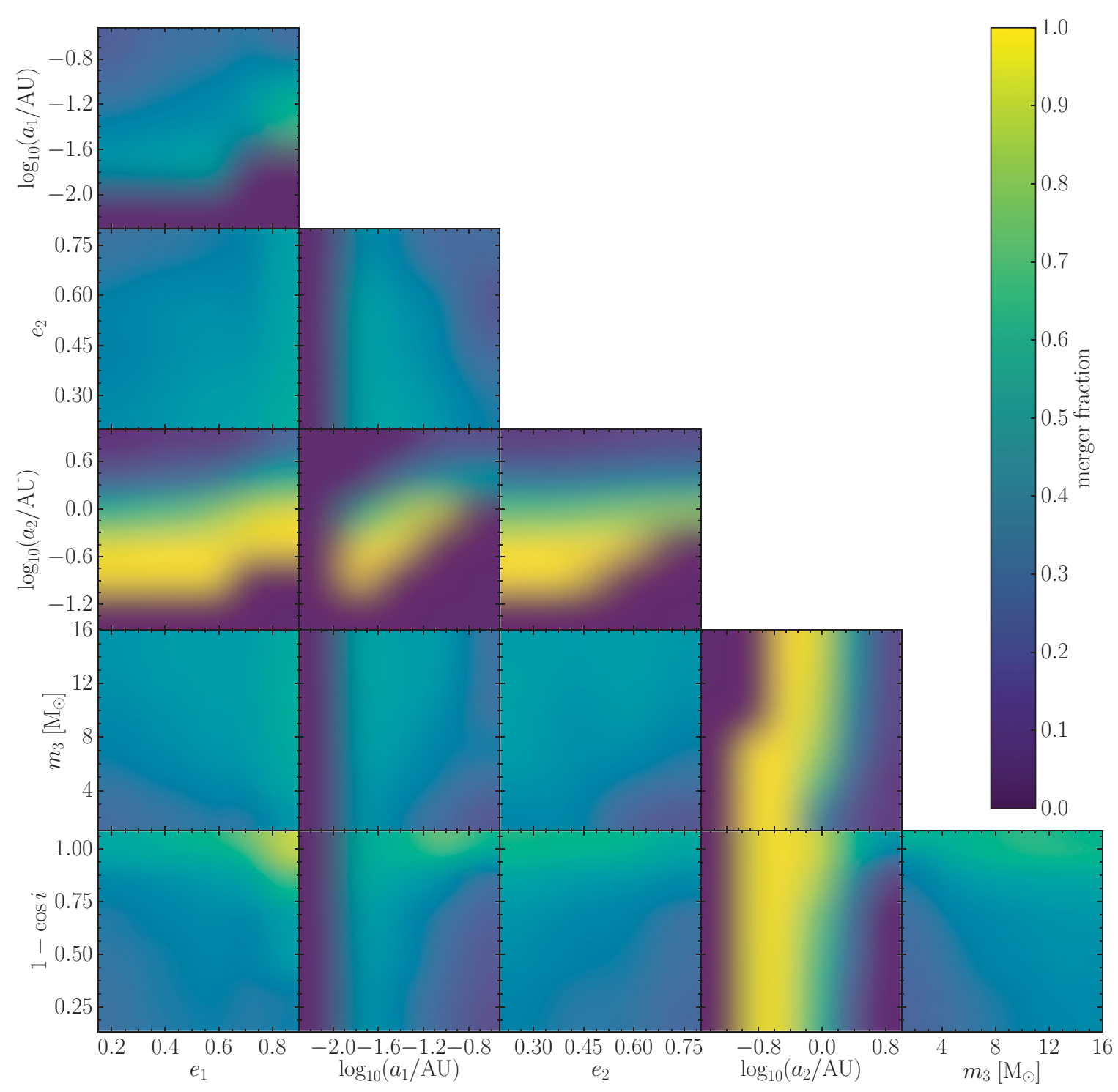

Figure A1. Merger fraction (colour-coded) as a function of various parameter pairs for the NSNS case with $m_{1}=1.6 \mathrm{M}_{\odot}, m_{2}=1.2$ $\mathrm{M}_{\odot}$, and $\left(g_{1}, g_{2}\right)=\left(180^{\circ}, 0^{\circ}\right)$. Panels represent $2 \mathrm{D}$ slices of the merger fraction of stable non-precessing triplets that would not merge within $10^{8} \mathrm{yr}$ as isolated binaries, but that do so as inner binaries of triplets because of the KL mechanism. We span the full range of possible combinations (see Table 3). From the plot, the parameter $a_{2}$ is the most important in shaping the value of the merger fraction (cf. green/yellow areas in the plots). A relevant role is also played by the relative inclination $i$, which at values close to $90^{\circ}$ triggers substantial KL oscillations. The sharp decreases (dark blue areas) occur instead because such points in the grid yield unstable or rapidly precessing systems, preventing or making pointless the corresponding simulations within our framework (see Section 3). 


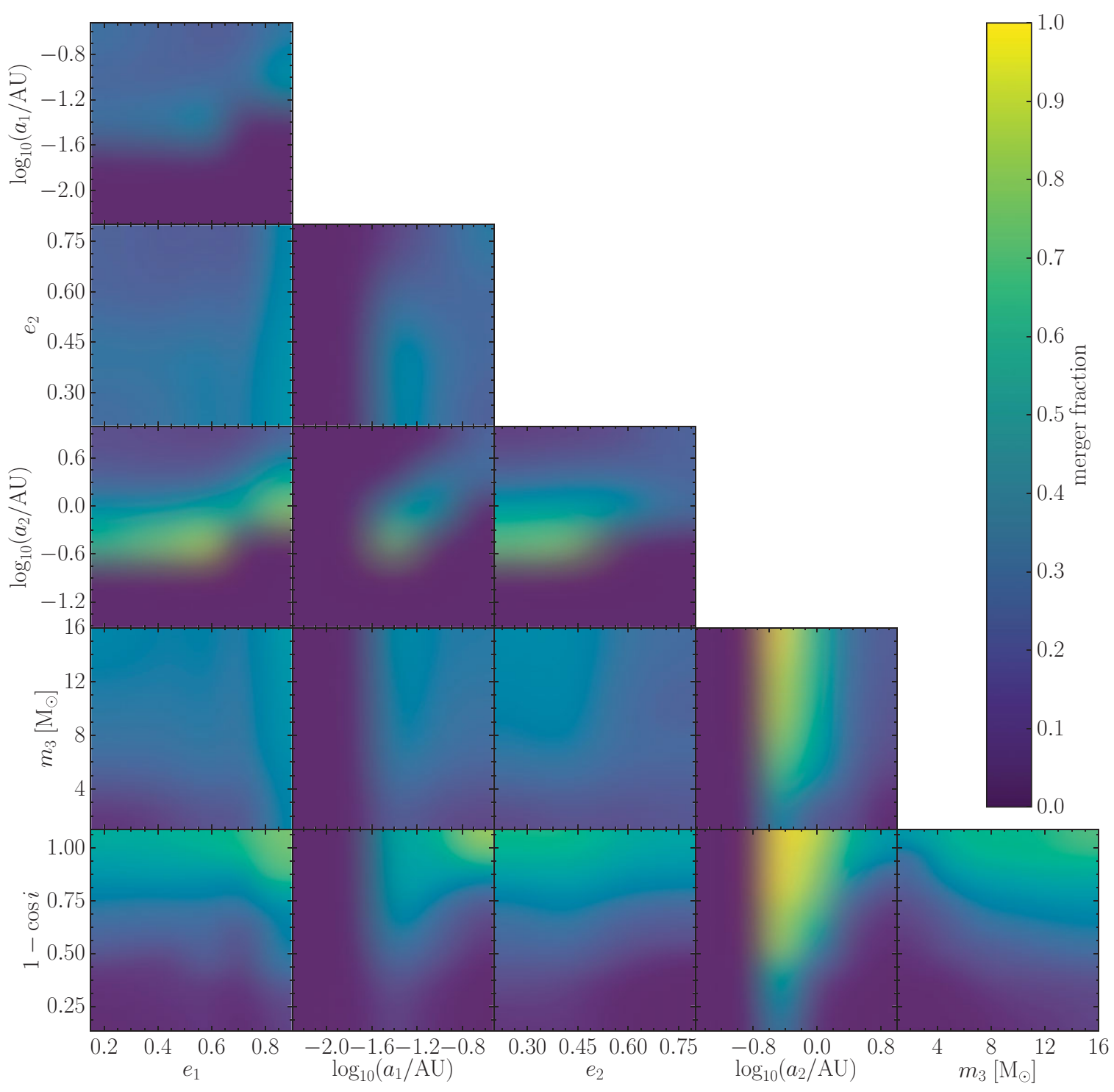

Figure A2. Same as Figure 7, but for the BHNS case with $m_{1}=15 \mathrm{M}_{\odot}$ and $m_{2}=1.8 \mathrm{M}_{\odot}$. 


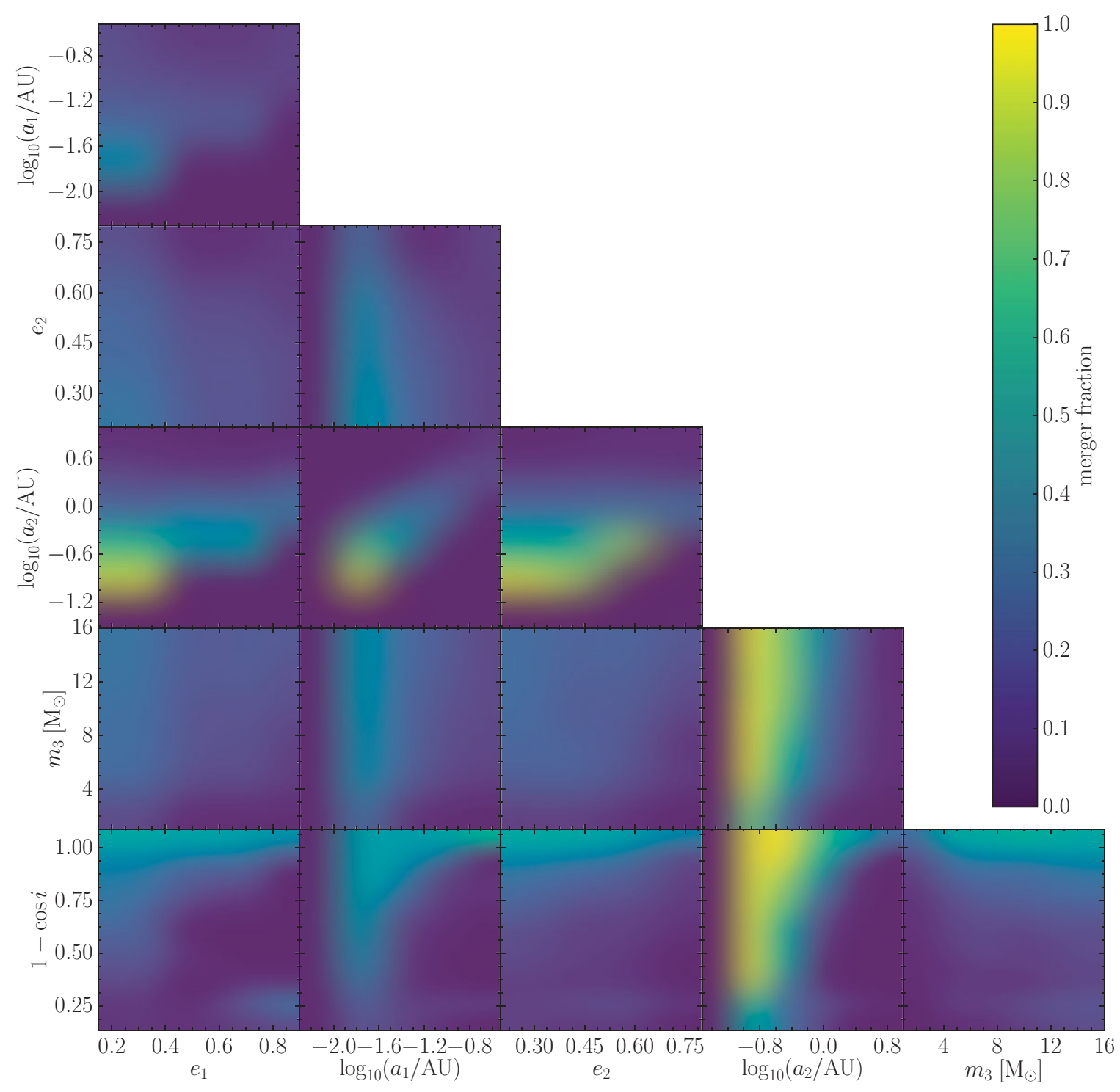

Figure A3. Same as Figure 7, but for the BHNS case with $m_{1}=7.5 \mathrm{M}_{\odot}, m_{2}=1.2 \mathrm{M}_{\odot}$, and $\left(g_{1}, g_{2}\right)=\left(90^{\circ}, 270^{\circ}\right)$. 